\title{
Evidence for a Magnetic-Field-Induced Ideal Type-II Weyl State in Antiferromagnetic Topological Insulator $\mathrm{Mn}\left(\mathrm{Bi}_{1-x} \mathrm{Sb}_{x}\right)_{2} \mathrm{Te}_{4}$
}

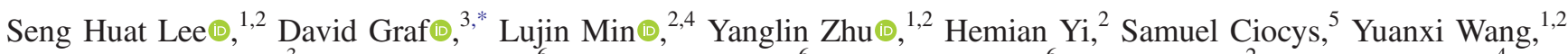 \\ Eun Sang Choi, ${ }^{3}$ Rabindra Basnet, ${ }^{6}$ Arash Fereidouni, ${ }^{6}$ Aaron Wegner $\odot,{ }^{6}$ Yi-Fan Zhao, ${ }^{2}$ Katrina Verlinde, ${ }^{4}$ \\ Jingyang He,${ }^{2,4}$ Ronald Redwing, ${ }^{1,4}$ V. Gopalan, ${ }^{4}$ Hugh O. H. Churchill, ${ }^{6}$ Alessandra Lanzara, ${ }^{5}$ \\ Nitin Samarth, ${ }^{1,2}$ Cui-Zu Chang, ${ }^{2}$ Jin Hu, ${ }^{6, \dagger}$ and Z. Q. Mao $\circledast^{1,2,4, \$}$ \\ ${ }^{1} 2 D$ Crystal Consortium, Materials Research Institute, The Pennsylvania State University, \\ University Park, Pennsylvania 16802, USA \\ ${ }^{2}$ Department of Physics, The Pennsylvania State University, University Park, Pennsylvania 16802, USA \\ ${ }^{3}$ National High Magnetic Field Lab, Tallahassee, Florida 32310, USA \\ ${ }^{4}$ Department of Materials Science and Engineering, The Pennsylvania State University, \\ University Park, Pennsylvania 16802, USA \\ ${ }^{5}$ Department of Physics, University of California, Berkeley, Berkeley, California 94720, USA \\ ${ }^{6}$ Department of Physics, University of Arkansas, Fayetteville, Arkansas 72701, USA
}

(Received 21 June 2020; revised 25 April 2021; accepted 8 June 2021; published 10 August 2021)

The discovery of Weyl semimetals (WSMs) has fueled tremendous interest in condensed matter physics. The realization of WSMs requires the breaking of either inversion symmetry (IS) or time-reversal symmetry (TRS). WSMs can be categorized into type-I and type-II WSMs, which are characterized by untilted and strongly tilted Weyl cones, respectively. Type-I WSMs with breaking of either IS or TRS and type-II WSMs with solely broken IS have been realized experimentally, but a TRS-breaking type-II WSM still remains elusive. In this article, we report transport evidence for a TRS-breaking type-II WSM observed in the intrinsic antiferromagnetic topological insulator $\mathrm{Mn}\left(\mathrm{Bi}_{1-x} \mathrm{Sb}_{x}\right)_{2} \mathrm{Te}_{4}$ under magnetic fields. This state is manifested by the electronic structure transition caused by the spin-flop transition. The transition results in an intrinsic anomalous Hall effect and negative $c$-axis longitudinal magnetoresistance attributable to the chiral anomaly in the ferromagnetic phases of lightly hole-doped samples. Our results establish a promising platform for exploring the underlying physics of the long-sought, ideal TRS-breaking type-II WSM.

DOI: 10.1103/PhysRevX.11.031032

\section{INTRODUCTION}

Weyl semimetals (WSMs) have become a forefront research topic in contemporary condensed matter physics [1-3]. They provide model platforms for studying concepts in high-energy physics, such as the magnetic chiral anomaly effect. Moreover, they provide a means of realizing potentially useful exotic quantum states of technological relevance, e.g., the quantum anomalous Hall insulator [4]. In WSMs, singly degenerate, linearly dispersed bands cross at Weyl nodes in momentum space; these nodes always appear in pairs with opposite chirality

\footnotetext{
*graf@magnet.fsu.edu

jinhu@uark.edu

zim1@psu.edu
}

Published by the American Physical Society under the terms of the Creative Commons Attribution 4.0 International license. Further distribution of this work must maintain attribution to the author(s) and the published article's title, journal citation, and DOI.
Subject Areas: Condensed Matter Physics,

Topological Insulators and can be understood as a monopole and an antimonopole of Berry flux. When the Weyl nodes are located at or near the chemical potential, their diverging Berry curvature can give rise to distinct properties such as a large intrinsic anomalous Hall effect (AHE) and an anomalous Nernst effect if time-reversal symmetry (TRS) is broken [5-8]. In addition, the bulk-edge correspondence principle leads WSMs to possess unique surface Fermi arcs [9-11], which can also generate exotic phenomena such as quantum oscillations arising from unconventional magnetic orbits $[12,13]$ and a bulk quantum Hall effect through the formation of Weyl orbits [14]. Further understanding of Weyl fermion physics requires an ideal WSM in which all Weyl nodes should be symmetry related and at the same energy level (at or near the chemical potential), without interference from any other bands $[1-3,15]$. However, although ideal Weyl states have been long pursued, they have been realized only in bosonic systems (i.e., photonic crystals [16]). An ideal fermionic WSM with distinct exotic properties is still lacking. The Weyl nodes of the WSMs discovered to date are either not close to the chemical 
potential, or not at the same energy level, or interfered with by other bands.

WSMs can be divided into two categories: type-I and type-II WSMs. Type-I WSMs, which feature untilted Weyl cones, can be further categorized into inversion-symmetrybreaking WSMs and TRS-breaking WSMs. The inversionsymmetry-breaking type-I WSM state was first discovered in TaAs-class materials [10,11,17-19], and the TRS-breaking type-I WSM was recently demonstrated in several magnetic materials such as GdPtBi [20], $\mathrm{Co}_{3} \mathrm{Sn}_{2} \mathrm{~S}_{2}$ [5,6,21-24], and $\mathrm{Co}_{2} \mathrm{MnGa}[25,26]$. Unlike type-I WSMs, type-II WSMs are characterized by strongly tilted Weyl cones that violate Lorentz invariance [27]. The inversion-symmetry-breaking type-II WSM was found in several nonmagnetic materials such as (W/Mo) $\mathrm{Te}_{2}$ [27-30], LaAlGe [31], and $\mathrm{TaIrTe}_{4}$ [32,33]; however, the TRSbreaking type-II WSM still remains elusive. $\mathrm{YbMnBi}_{2}$ has been claimed to be a member of this category [34], but it is debated whether it breaks TRS [35]. In this article, we report experimental evidence for a recently predicted, magneticfield-induced ideal TRS-breaking type-II Weyl state with only one pair of Weyl nodes in $\mathrm{MnBi}_{2} \mathrm{Te}_{4}$ [36,37].

$\mathrm{MnBi}_{2} \mathrm{Te}_{4}$ has been demonstrated as the first intrinsic antiferromagnetic (AFM) topological insulator (TI) [3638]. The combination of spontaneous magnetization and nontrivial band topology in this material allows access to a variety of exotic topological quantum states in its $2 \mathrm{D}$ thin layers, including the quantum anomalous Hall insulator [39], the axion insulator [40], and the $C=2$ Chern insulator [41]. In addition, $\mathrm{MnBi}_{2} \mathrm{Te}_{4}$ is also predicted to host a long-sought, ideal type-II TRS-breaking WSM when its AFM order is coerced into FM order by a magnetic field parallel to the $c$ axis [36,37]. Theoretical studies show that the interlayer hybridization, combined with the protection of $C_{3}$ rotational symmetry, induces band crossings in the FM phase, resulting in a single pair of strongly tilted, typeII Weyl cones with the Weyl nodes above the chemical potential $[36,37]$. Here, we provide transport evidence for such an ideal WSM state using magnetotransport measurements in $\mathrm{Mn}\left(\mathrm{Bi}_{1-x} \mathrm{Sb}_{x}\right)_{2} \mathrm{Te}_{4}$. By tuning the $\mathrm{Sb}$ : Bi ratio, we observe an electronic structure transition driven by the spinflop transition. The transition leads to an AHE that we attribute to an intrinsic origin and a large negative $c$-axis longitudinal magnetoresistance that is consistent with the chiral anomaly. All these results provide strong support for the existence of the ideal Weyl state in the FM phase of this material.

\section{RESULTS AND DISCUSSION}

Prior studies of $\mathrm{Mn}\left(\mathrm{Bi}_{1-x} \mathrm{Sb}_{x}\right)_{2} \mathrm{Te}_{4}[42,43]$ show that the carrier density is minimized near $x \sim 0.3$, where the carrier type also changes from electron to hole. Although there is only a small difference between the Néel temperatures $\left(T_{\mathrm{N}}\right)$ of $\mathrm{MnBi}_{2} \mathrm{Te}_{4}\left(T_{\mathrm{N}}=25 \mathrm{~K}\right)$ and $\mathrm{MnSb}_{2} \mathrm{Te}_{4}\left(T_{\mathrm{N}}=19 \mathrm{~K}\right)$ $[42,43]$, Sb substitution for Bi induces striking changes in the magnetic properties of the $\mathrm{Bi}$ compound. Undoped $\mathrm{MnBi}_{2} \mathrm{Te}_{4}$ undergoes a spin-flop transition upon increasing

TABLE I. Information of the $\mathrm{Mn}\left(\mathrm{Bi}_{1-x} \mathrm{Sb}_{x}\right)_{2} \mathrm{Te}_{4}$ samples used in this study, including Sb content $x$, carrier type, $\mathrm{SdH}$ oscillation frequency $f_{\mathrm{osc}}$, and carrier densities estimated from Hall coefficient and $f_{\text {osc }}$. The Hall carrier density is extracted from the linear background of $\rho_{y x}$ in FM phase at 2 or $0.7 \mathrm{~K}$. For the lightly hole-doped samples $\left(S 3 B\right.$ and $S 3 C$ ) which exhibit strong nonlinear field dependence in $\rho_{y x}$ due to the coexistence of electron and hole pockets, the Hall carrier densities given in the table represent the difference between the hole and electron density, $n_{h}-n_{e}$, estimated from the slope of $\rho_{y x}$ in the high field limit (approximately $40 \mathrm{~T}$ ), where $\rho_{y x} \approx\left[B /\left(n_{h}-n_{e}\right) e\right]$ (see the text).

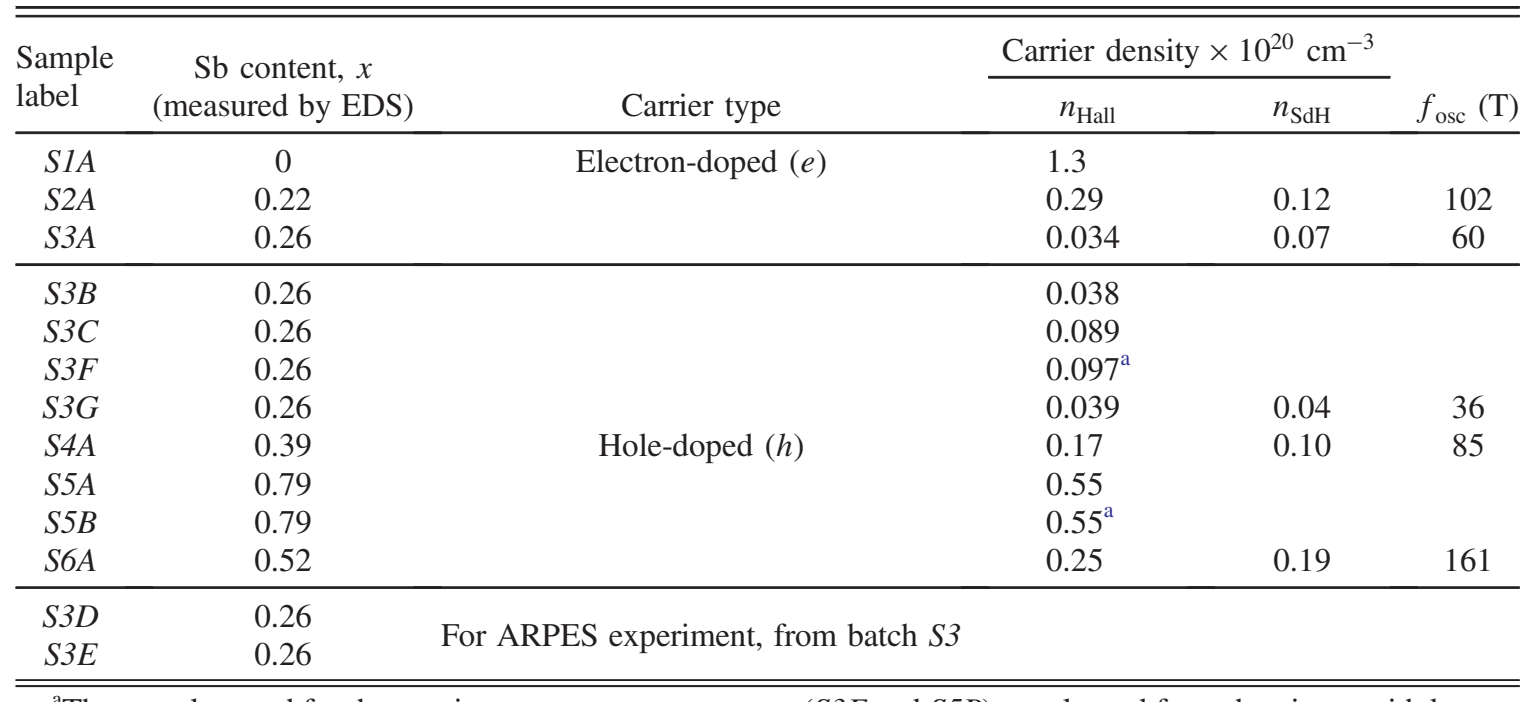

${ }^{\mathrm{a}}$ The samples used for the $c$-axis transport measurements $(S 3 F$ and $S 5 B)$ are cleaved from the pieces with known Hall carrier densities. 


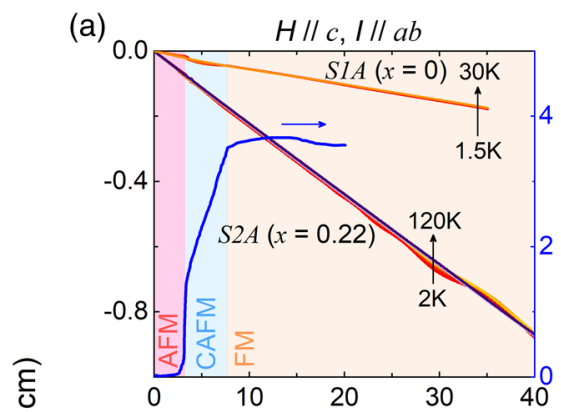

(b)

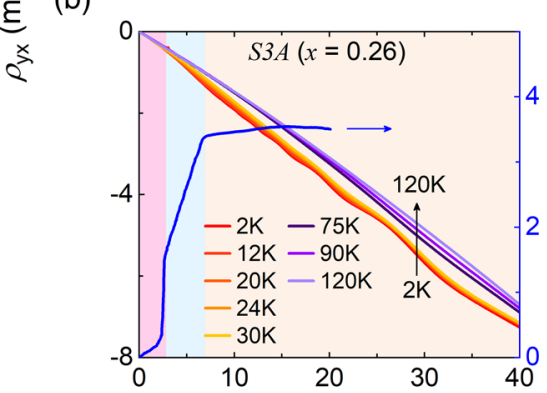

(g)

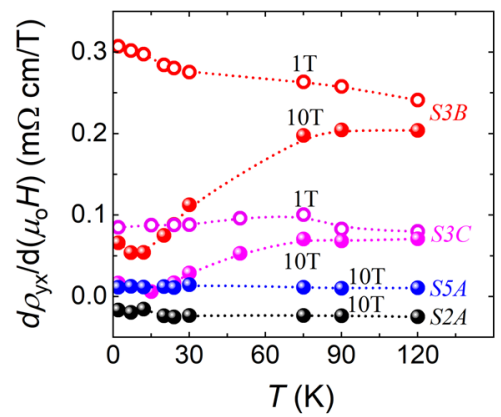

(c)

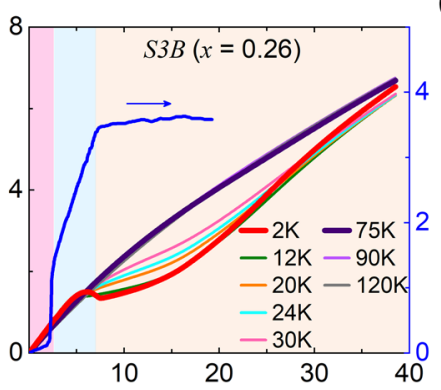

(d)

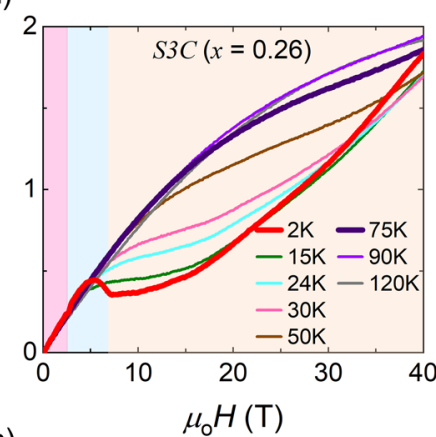

(h)

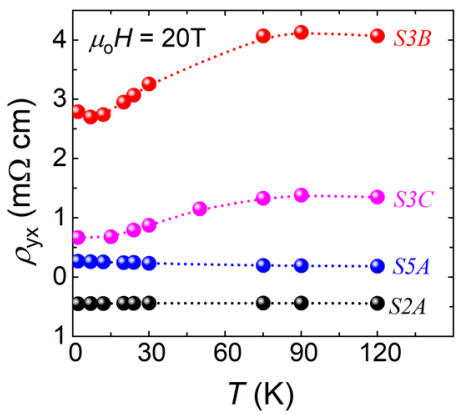

(e)

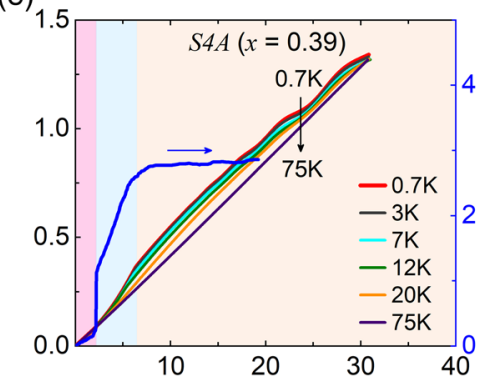

(f)

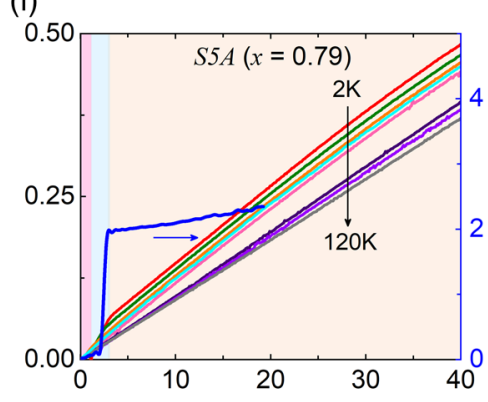

(i)

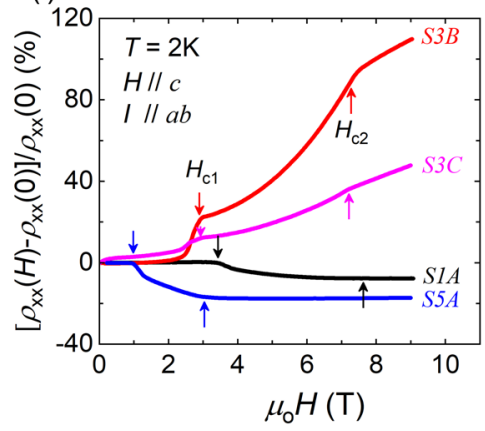

FIG. 1. Magnetic and transport properties of $\mathrm{Mn}\left(\mathrm{Bi}_{1-x} \mathrm{Sb}_{x}\right)_{2} \mathrm{Te}_{4}$. Magnetic field dependence of Hall resistivity $\rho_{y x}$ at various temperatures and magnetization $M$ at $2 \mathrm{~K}$ for $\mathrm{Mn}\left(\mathrm{Bi}_{1-x} \mathrm{Sb}_{x}\right)_{2} \mathrm{Te}_{4}$ with (a) $x=0$ and 0.22 , (b)-(d) 0.26 , (e) 0.39 , and (f) 0.79. All these measurements are conducted with the currents applied along the $a b$ plane and the magnetic fields perpendicular to the plane. The rose, blue, and orange regions refer to the AFM, CAFM, and FM phase regions, respectively, determined by magnetization [blue curves in (a)-(f)]. The heavily (a),(f) and moderately (e) doped samples exhibit linear magnetic field dependence for $\rho_{y x}$ or almost linear $\rho_{y x}$ background, while the lightly doped samples $S 3 A, S 3 B$, and $S 3 C$ show nonlinear field dependence in $\rho_{y x}$ (b)-(d). The $S 3 A, S 3 B$, and $S 3 C$ samples used in this study are taken from the same growth batch but display either electronlike (b) or holelike (c),(d) transport behavior in $\rho_{y x}(H)$ owing to inevitable chemical inhomogeneity as discussed in the text. (g) Temperature dependences of the Hall resistivity slope $d \rho_{y x} / d\left(\mu_{o} H\right)$ at 1 and $10 \mathrm{~T}$ for the lightly hole-doped (S3B, S3C), heavily electron-doped (S2A), and heavily hole-doped (S5A) samples. (h) Temperature dependence of Hall resistivity $\rho_{y x}$ at $20 \mathrm{~T}$ for the lightly hole-doped ( $S 3 B$ and $S 3 C$ ), heavily electron-doped (S2A), and heavily hole-doped (S5A) samples. (i) The transverse in-plane magnetoresistivity for lightly hole-doped ( $S 3 B$ and $S 3 C$ ), heavily electron-doped (S1A), and heavily hole-doped samples (S5A). The downward and upward arrows refer to the two magnetic transitions upon increasing the magnetic field along the $c$ axis, i.e., the AFM-to-CAFM transition at $H_{c 1}$ and the CAFM-to-FM transition at $H_{c 2}$.

the magnetic field along the $c$ axis, manifested by the AFM-to-canted-antiferromagnetic (CAFM) transition at $H_{c 1}$ and the CAFM-to-FM transition at $H_{c 2}$ [44]. Both $H_{c 1}$ and $H_{c 2}$ are suppressed by increasing Sb content and tend to merge as $x$ approaches 1 [43]. Moreover, the $\mathrm{Sb}$ substitution for $\mathrm{Bi}$ also leads to a nearly linear decrease of the Mn saturation moment, from $3.56 \mu_{\mathrm{B}} / \mathrm{Mn}$ for $\mathrm{MnBi}_{2} \mathrm{Te}_{4}$ to $1.57 \mu_{\mathrm{B}} / \mathrm{Mn}$ for $\mathrm{MnSb}_{2} \mathrm{Te}_{4}$ [43]. This result can be attributed to magnetic fluctuations arising from competing magnetic interactions. There are two possible origins of competing magnetic interactions. One is the competition between the dominant intralayer FM interaction and the direct AFM exchange interaction of the nearest Mn-Mn neighbors [43]. The other is the competition between the interlayer AFM interaction and the ferrimagnetic order mediated by the $\mathrm{Mn}-(\mathrm{Bi}, \mathrm{Sb})$ antisite defects [45-47]. In this article, we focus on investigating the magnetotransport properties of $\mathrm{Mn}\left(\mathrm{Bi}_{1-x} \mathrm{Sb}_{x}\right)_{2} \mathrm{Te}_{4}$ under high magnetic 
fields (up to $41.5 \mathrm{~T}$ ) and seek transport evidence for the predicted type-II WSM.

Single crystals of $\mathrm{Mn}\left(\mathrm{Bi}_{1-x} \mathrm{Sb}_{x}\right)_{2} \mathrm{Te}_{4}$ used in this work are grown by a flux method and characterized using x-ray diffraction (see Sec. III). The composition analyses using energy-dispersive x-ray spectroscopy (EDS) show that the measured $\mathrm{Sb}$ content $x$ deviates from the nominal value. The measured $x$ values for each batch of samples as well as other sample information are given in Table I. EDS analyses also show that the variation of the $\mathrm{Bi} / \mathrm{Sb}$ ratio between different spots of an identical crystal is small, with the largest difference being about 2.2\%. We do not observe any clustering of $\mathrm{Bi}$ and $\mathrm{Sb}$ in the EDS mapping, suggesting that both $\mathrm{Bi}$ and $\mathrm{Sb}$ distributions are relatively homogenous; Supplemental Material, Sec. S1 [48], shows representative data collected on a sample from the $x=0.26$ batch. From isothermal magnetization measurements at $2 \mathrm{~K}$ [blue curves in Figs. 1(a)-1(f)], we find that the evolution of magnetism of $\mathrm{Mn}\left(\mathrm{Bi}_{1-x} \mathrm{Sb}_{x}\right)_{2} \mathrm{Te}_{4}$ with $\mathrm{Sb}$ content is consistent with prior studies $[42,43]$. The AFM, CAFM, and FM phases determined by the magnetization data for each sample are color coded in Figs. 1(a)-1(f). We conduct high magnetic field Hall resistivity $\rho_{y x}$ measurements for representative samples with various $x$ and find that the magnetic field and temperature dependences of $\rho_{y x}$ exhibit drastic changes with increasing $\mathrm{Sb}$ content. The slope of $\rho_{y x}$ is negative for $x<0.26$ [Fig. 1(a)] but positive for $x>0.26$ [Figs. 1(e) and 1(f)], indicating that $x=0.26$ is the critical concentration for the carrier type change [Figs. 1(b)-1(d)].

As shown in Figs. 1(b)-1(d), while samples $S 3 A, S 3 B$, and $S 3 C$ are all taken from the same batch, sample $S 3 A$ is lightly electron doped, but samples $S 3 B$ and $S 3 C$ are lightly hole doped. Such strong sample dependences of transport properties, which are not observed in $x>0.26$ (or $<0.26$ ) samples, can be attributed to the fact that the $x=0.26$ samples have chemical potentials close to the band edge, which makes their magnetotransport properties extremely sensitive to the electronic structure transition induced by the spin-flop transition, as discussed below.

To locate the position of the chemical potentials in the $x=0.26$ samples, we perform angle-resolved photoemission spectroscopy (ARPES) measurements (see Sec. III) on two samples $(S 3 D$ and $S 3 E$ ) selected from the $x=0.26$ batch along the $\Gamma-K$ direction and find that their chemical potentials are indeed close to the conduction or valence band edges. Samples $S 3 D$ and $S 3 A$ are originally from the same piece. As shown in Fig. 2(a)(i), the chemical potential of sample $S 3 D$ is close to the bottom of the bulk conduction band, in good agreement with the light electron doping probed in the transport measurements of sample $S 3 A$ (see Table I). However, for sample $S 3 E$, we measure two spots and find that its chemical potential is pushed into the bulk gap at spot 1 [Fig. 2(a)(ii)] but near the top of the bulk valence band at spot 2 [Fig. 2(a)(iii)]. For spot 2, we also construct constant energy maps within two different energy
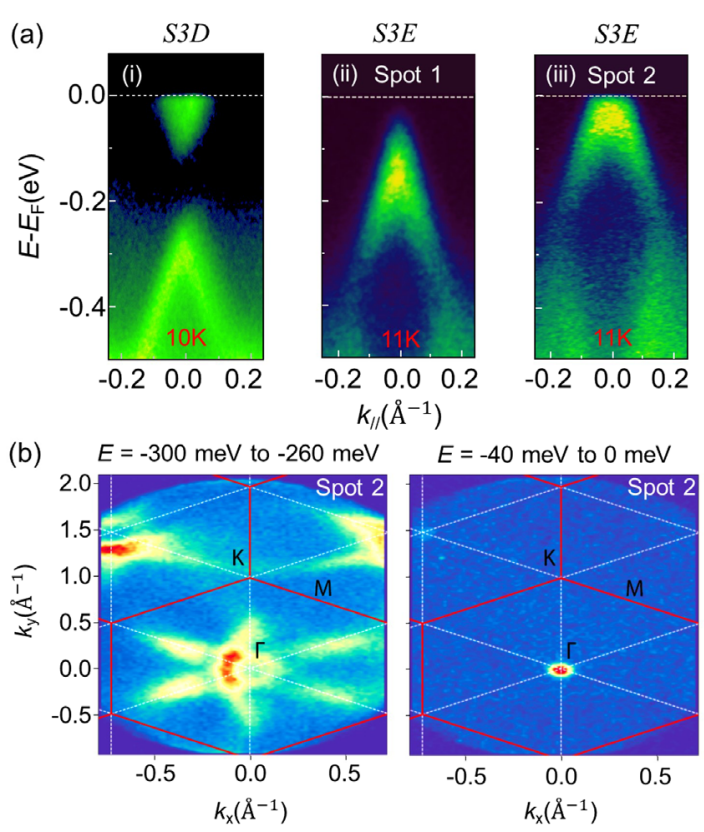

FIG. 2. Band structures of $\mathrm{Mn}\left(\mathrm{Bi}_{1-x} \mathrm{Sb}_{x}\right)_{2} \mathrm{Te}_{4}$. (a) ARPES spectra measured on (i) sample $S 3 D$ using the photon energy of $21.218 \mathrm{eV}$ and (ii),(iii) sample $S 3 E$ using the photon energy of $81 \mathrm{eV}$. Spectra in (ii) and (iii) are measured on two different spots of sample $S 3 E$. All the spectra in (a) are measured below $T_{\mathrm{N}}=$ $25 \mathrm{~K}$ along the $\Gamma-K$ direction [see (b)]. (b) ARPES constant energy maps at spot 2 , acquired by integrating over -300 to $-260 \mathrm{meV}$ (left) and -40 to $0 \mathrm{meV}$ (right). A pointlike Fermi surface centered at $\Gamma$ can be seen on the $k_{x}-k_{y}$ plane.

windows ( -300 to $-260 \mathrm{meV}$ and -40 to $0 \mathrm{meV}$ ) [Fig. 2(b)], showing that the projection of the Fermi surface on the $k_{x}-k_{y}$ plane is centered at the $\Gamma$ point. Since ARPES is a surface-sensitive probe, the difference of the ARPES spectra between spots 1 and 2 does not necessarily imply that the bulk involves large electronic inhomogeneity. If the measured sample is bulk insulating, small doping differences at the surface from surface adsorbents can cause large changes to the Fermi level at the surface. This phenomenon is often seen in topological insulators (e.g., see Ref. [49]). Given that sample $S 3 E$ is taken from the $x=0.26$ batch and shows the chemical potential inside the gap at spot 1, its bulk is either insulating or lightly doped. As such, the ARPES spectra difference between spot 1 and 2 in Figs. 2(a)(ii) and 2(a)(iii) is most likely attributable to the small doping difference induced by surface adsorbents. This conjecture is verified by transport measurements on samples $S 3 B$ and $S 3 C$, which are also from the $x=0.26$ batch. From the carrier density derived from the Hall resistivity data of the paramagnetic (PM) state at $75 \mathrm{~K}$ (see Fig. 3), the Fermi wave vector $k_{\mathrm{F}}$ is estimated to be approximately 0.012 and $0.015 \AA^{-1}$ for samples $S 3 B$ and $S 3 C$, respectively, indicating that the average chemical potential of these samples is much closer to the top of the bulk valence band as compared to the scenario seen in Fig. 2(a)(iii). These lightly hole-doped 


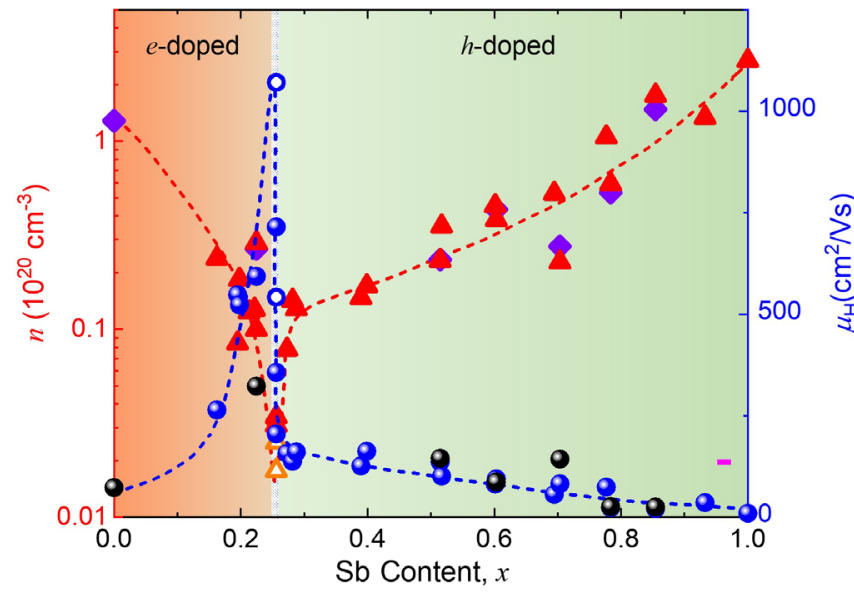

FIG. 3. Composition dependence of carrier density $n$ and transport mobility $\mu_{\mathrm{H}}$ of $\mathrm{Mn}\left(\mathrm{Bi}_{1-x} \mathrm{Sb}_{x}\right)_{2} \mathrm{Te}_{4}$. For heavily and moderately doped samples showing linear or almost linear $\rho_{y x}$ (Fig. 1 and Supplemental Material, Fig. S1 [48]), $n$ is obtained from the linear slope of $\rho_{y x}(H)$ at $T=2 \mathrm{~K}$ for the FM phase (purple diamond) and $75 \mathrm{~K}$ for the PM phase (red triangle). The black and blue circles represent transport mobility $\mu_{\mathrm{H}}[=$ $\left.R_{\mathrm{H}} / \rho_{x x}(0)\right]$ for the FM and PM phases, respectively. For lightly hole-doped samples (from the $x=0.26$ batch) with nonlinear $\rho_{y x}(H), n$ (empty orange triangle) and $\mu_{\mathrm{H}}$ (empty blue circle) are extracted from the two-band model fit (see Supplemental Material, Sec. S2 [48]), and only the data of dominant bands are presented here. The magenta bar represents the maximal statistical error of the measured $\mathrm{Sb}$ content by EDX.

samples enable us to observe remarkable signatures of the predicted Weyl state, as presented below.

We use the magnetic field dependence of $\rho_{y x}$ to estimate the carrier densities of the samples shown in Fig. 1. The results of this analysis are summarized in Fig. 3; note that the red triangles and purple diamonds in Fig. 3 represent the carrier densities of the samples in the PM states at $75 \mathrm{~K}$ and FM states at $2 \mathrm{~K}$, respectively (see more $\rho_{y x}$ data of additional samples in Supplemental Material, Fig. S1 [48]). The heavily electron-doped (S1A) or hole-doped $(S 5 A)$ samples exhibit a linear magnetic field dependence of $\rho_{y x}$ in both the FM and PM phases [Figs. 1(a) and 1(f)]. The moderately doped samples ( $S 2 A$ and $S 4 A$ ) exhibit quantum oscillations in $\rho_{y x}$ in the FM phase, with their background of $\rho_{y x}$ showing slight deviation from the linear field dependence [Fig. 1(e)]. The carrier densities of these heavily or moderately doped samples are, thus, estimated directly using the linear slope of $\rho_{y x}$ (i.e., Hall coefficient $R_{\mathrm{H}}$ ); this estimation shows little or no variation between the PM and FM states (Fig. 3). In strong contrast, in the lightly hole-doped samples $S 3 B$ and $S 3 C$ [Figs. 1(c) and 1(d)], $\rho_{y x}$ has a distinct nonlinear dependence on the magnetic field in both PM and FM phases. In these cases, we estimate the carrier densities through the two-band model fits for their PM states (see Supplemental Material, Sec. S2 [48]). For their FM phases which are characterized by the coexistence of electron and hole pockets as discussed below, we extract the difference between the hole and electron density, $n_{h}-n_{e}$, from the slope of $\rho_{y x}$ in the high field limit (approximately $40 \mathrm{~T}$ ), where $\rho_{y x} \approx\left[B /\left(n_{h}-n_{e}\right) e\right]$ according to the two-band model (see below). We include these data as well as the carrier densities of the FM phases of other samples in Table I. All the carrier densities mentioned below refer to the Hall carrier density of the PM phases (Fig. 3) unless specified. Figure 3 shows that the $x \sim 0.26$ samples have a distinctly lower carrier density at their PM phases compared to other compositions; the minimum is approximately $2 \times 10^{18} \mathrm{~cm}^{-3}$, about 2 orders of magnitude smaller than that of $\mathrm{MnBi}_{2} \mathrm{Te}_{4}$ [44,50]. Furthermore, we find that the samples with lower carrier density have higher carrier transport mobility $\mu_{\mathrm{H}}$. The maximum value of $\mu_{\mathrm{H}}$ is approximately $1.1 \times 10^{3} \mathrm{~cm}^{2} / \mathrm{Vs}$ (see Sec. III), about 2 orders of magnitude larger than in $\mathrm{MnBi}_{2} \mathrm{Te}_{4}$ (approximately $58 \mathrm{~cm}^{2} / \mathrm{Vs}$ ) and $\mathrm{MnSb}_{2} \mathrm{Te}_{4}\left(9.7 \mathrm{~cm}^{2} / \mathrm{Vs}\right)$ (Fig. 3).

The access to the lower carrier density in $\mathrm{Mn}\left(\mathrm{Bi}_{1-x} \mathrm{Sb}_{x}\right)_{2} \mathrm{Te}_{4}$ enables the observation of the transport signatures of the predicted ideal FM WSM. As noted above, it has been theoretically predicted that the AFM-toFM transition driven by magnetic fields along the $c$ axis should lead to a type-II Weyl state for which Weyl nodes are at the touching points of electron and hole pockets [36,37]. As such, for lightly hole-doped samples, we expect a transition from an electronic state with only a hole pocket in the AFM phase to the one with the coexistence of electron and hole pockets in the FM phase. In the $\rho_{y x}$ measurements of samples $S 3 B$ and $S 3 C$, we do observe evidence for such a transition. As shown in Figs. 1(c) and 1(d), the field dependences of $\rho_{y x}$ of these samples exhibit signatures of hole doping and are nearly temperature independent in their PM states above $75 \mathrm{~K}$. However, when the temperature is lowered below $75 \mathrm{~K}$, their $\rho_{y x}$ values reduce significantly, and the values of $\rho_{y x}$ of the FM phase at $2 \mathrm{~K}$ are much smaller than those of the PM states above $75 \mathrm{~K}$ in a wide field range above $H_{c 2}$. We plot the variation of $\rho_{y x}$ with the temperature at $20 \mathrm{~T}$ in Fig. 1(h), where a drastic decrease of $\rho_{y x}$ from the PM to the FM state is seen clearly in samples $S 3 B$ and $S 3 C$, in stark contrast with the very small or negligible variation of $\rho_{y x}$ with temperature seen in moderately and heavily doped samples (e.g., $S 2 A$ [Fig. 1(a)] and S5A [Fig. 1(f)]). Moreover, the slopes of $\rho_{y x}$ of samples $S 3 B$ and $S 3 C$ exhibit striking decreases from the PM to FM states but small increases from the PM to AFM states, sharply contrasted with heavily doped samples for which $d \rho_{y x} / d\left(\mu_{\mathrm{o}} H\right)$ hardly changes across the PM-FM transition, as shown in Fig. 1(g), which plots $d \rho_{y x} / d\left(\mu_{\mathrm{o}} H\right)$ at 10 and $1 \mathrm{~T}$ as a function of the temperature for samples $S 3 B, S 3 C, S 2 A$, and $S 5 A$. The drastic decreases of $\rho_{y x}$ as well as the corresponding variation of $d \rho_{y x} / d\left(\mu_{\mathrm{o}} H\right)$ from the PM to the FM state in samples $S 3 B$ and $S 3 C$ can be attributed to the presence of 
an electron pocket in the FM state, since electrons lead to negative Hall resistivity. That is, the lightly hole-doped samples should feature the coexistence of electron and hole pockets in their FM phases. Note that, while the $T_{\mathrm{N}}$ of the AFM phase is approximately $25 \mathrm{~K}$, the ordering temperature of the field-driven FM phase should be beyond $25 \mathrm{~K}$, since strong magnetic fields can force a PM state to an FM state at temperatures not far away from $T_{\mathrm{N}}$. This result explains why $\rho_{y x}$ decrease starting from $75 \mathrm{~K}$ for samples $S 3 B$ and $S 3 C$. From Figs. 1(c) and 1(d), we can also see that the discrepancy of $\rho_{y x}$ between the PM and FM states tends to diminish in the high field limit. This tendency is because $\rho_{y x}$ in the high field limit resumes a single-band behavior, i.e., showing linear field dependence [i.e., $\rho_{y x} \propto B /\left(n_{h}-n_{e}\right)$ ] for noncompensated systems; this result can be derived from the two-band model for Hall resistivity, $\rho_{y x}=\left\{\left[\left(n_{h} \mu_{h}^{2}-n_{e} \mu_{e}^{2}\right)+\right.\right.$ $\left.\left.\mu_{h}^{2} \mu_{e}^{2}\left(n_{h}-n_{e}\right) B^{2}\right] /\left[\left(n_{e} \mu_{e}+n_{h} \mu_{h}\right)^{2}+\mu_{h}^{2} \mu_{e}^{2}\left(n_{h}-n_{e}\right)^{2} B^{2}\right]\right\}$. $(B / e)$.

The electronic structure transition concurrent with the spin-flop transition is also manifested by the anomalous peak of $\rho_{y x}$ present in the CAFM state [see the red curves in Figs. 1(c) and 1(d)], which indicates that the electron pockets start to emerge in the CAFM state. The in-plane transverse magnetoresistivity $\mathrm{MR}=\left[\rho_{x x}(H)-\rho_{x x}(0)\right] /$ $\rho_{x x}(0)$ measured on samples $S 3 B$ and $S 3 C$ provides further support for the electronic structure transition driven by the spin-flop transition. As shown in Fig. 1(i), the spin-flop transitions in these two samples lead to positive MR (e.g., approximately $110 \%$ at $9 \mathrm{~T}$ and $2 \mathrm{~K}$ for sample $S 3 B$ ), sharply contrasted with the heavily electron- and holedoped samples $S 1 A$ and $S 5 A$, whose spin-flop transitions result in the negative MR due to the suppression of magnetic scattering by magnetic fields. This contrast suggests that the spin-flop transition in the lightly holedoped samples results in an additional large positive term in MR, which can be attributed to the sharp increase of carrier mobility due to the presence of Weyl nodes near the Fermi level, as discussed below. Furthermore, the electronic structure transition driven by the spin-flop transition is also evidenced by the temperature dependence of quantum oscillation frequency (see below).

If this implicit electronic structure transition leads to an FM WSM as the theory predicts [36,37], transport measurements should reveal the characteristic transport signatures such as a large intrinsic AHE and a pronounced negative MR due to the chiral anomaly. In our experiments, we indeed observe magnetotransport signatures that are (a)
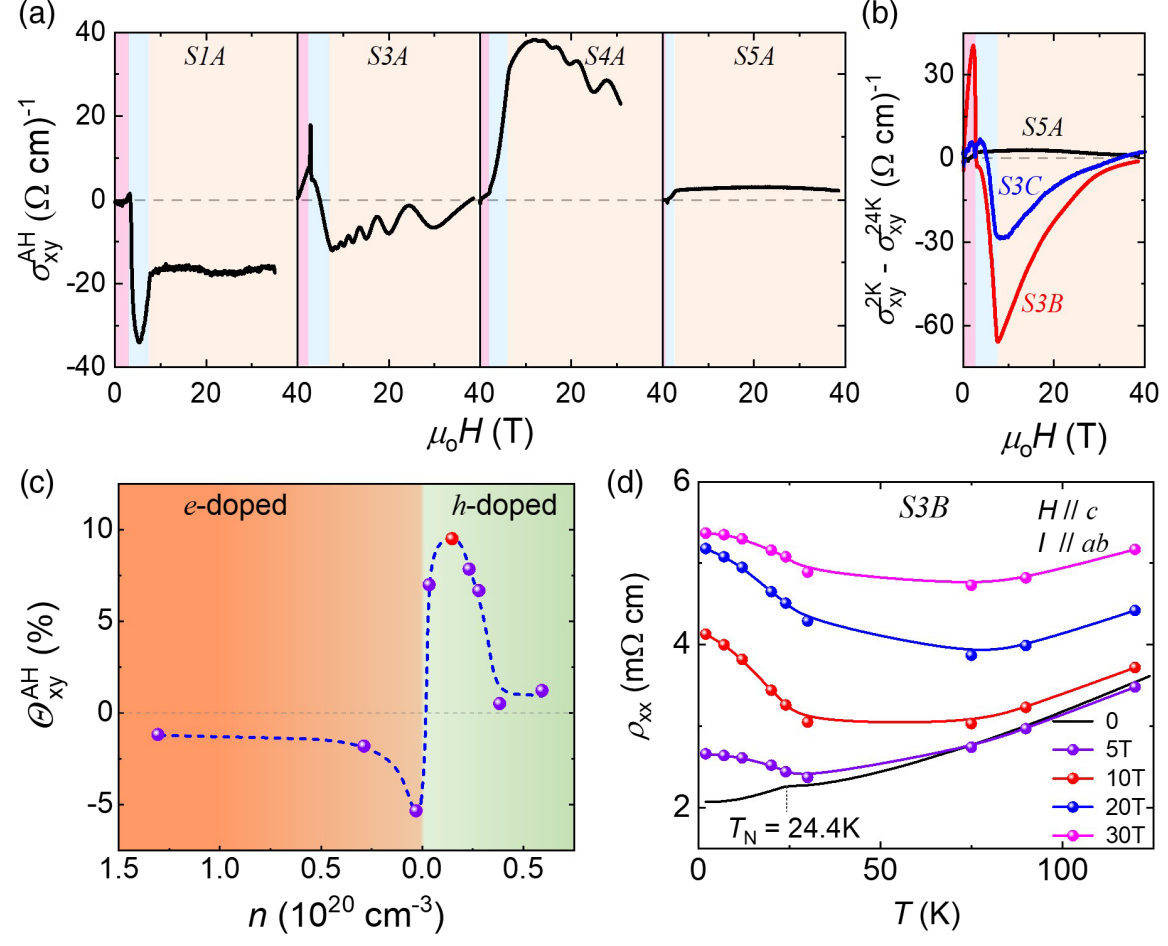

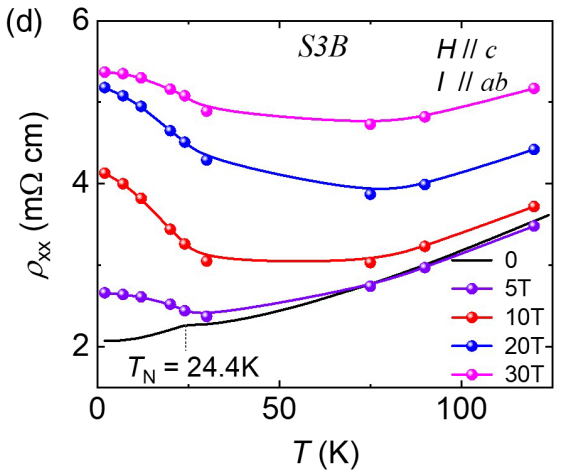

FIG. 4. Anomalous Hall effect in $\mathrm{Mn}\left(\mathrm{Bi}_{1-x} \mathrm{Sb}_{x}\right)_{2} \mathrm{Te}_{4}$. (a) Field dependence of anomalous Hall conductivity $\sigma_{x y}^{\mathrm{AH}}$ for samples $S 1 A, S 3 A$, $S 4 A$, and $S 5 A$ at 2 and 0.7 K. $\sigma_{x y}^{\mathrm{AH}}$ is derived using $\sigma_{x y}^{\mathrm{AH}}=\rho_{y x}^{\mathrm{AH}} /\left[\rho_{x x}^{2}+\rho_{y x}^{2}\right]$, where $\rho_{y x}^{\mathrm{AH}}$ is the anomalous Hall resistivity, obtained after subtracting the normal Hall contribution $\left(\rho_{y x}^{\mathrm{N}}=R_{\mathrm{H}} H\right)$ (see Sec. III). (b) Field dependence of $\sigma_{x y}^{2 \mathrm{~K}}-\sigma_{x y}^{24 \mathrm{~K}}$ for $S 3 B, S 3 C$, and $S 5 A$. $\sigma_{x y}^{2 \mathrm{~K}}$ and $\sigma_{x y}^{24 \mathrm{~K}}$ are extracted using $\sigma_{x y}=\rho_{y x} /\left(\rho_{x x}^{2}+\rho_{y x}^{2}\right)$ at 2 and $24 \mathrm{~K}$, respectively. (c) The maximal anomalous Hall angle $\Theta_{x y}^{\mathrm{AH}}=\sigma_{x y}^{\mathrm{AH}} / \sigma_{x x}$ in the FM phase as a function of the carrier density, obtained from the field dependence of $\Theta_{x y}^{\mathrm{AH}}$ shown in Supplemental Material, Fig. S3 [48]. All data points are obtained at $2 \mathrm{~K}$, except for $S 4 A\left(0.7 \mathrm{~K}\right.$, red circle). (d) Temperature dependence of longitudinal resistivity $\rho_{x x}$ for $S 3 B$ at various fields (applied along the $c$ axis). 
consistent with these effects in the lightly and moderately hole-doped samples. As indicated above, a large intrinsic AHE of an FM WSM arises from the diverging Berry curvature of the Weyl nodes, which is manifested by large intrinsic anomalous Hall conductivity $\sigma_{x y}^{\mathrm{AH}}$ and anomalous Hall angle $\left(\Theta_{x y}^{\mathrm{AH}}=\sigma_{x y}^{\mathrm{AH}} / \sigma_{x x}\right)$. Figure $4(\mathrm{a})$ presents $\sigma_{x y}^{\mathrm{AH}}$ at $T=2$ or $0.7 \mathrm{~K}$ for the heavily and lightly electron-doped samples ( $S 1 A$ and $S 3 A$ ) and moderately and heavily holedoped samples ( $S 4 A$ and $S 5 A$ ). These data are derived from $\rho_{y x}$ and longitudinal resistivity $\rho_{x x}$ (Supplemental Material, Fig. S2 [48]) via tensor conversion, i.e., $\sigma_{x y}^{\mathrm{AH}}=\rho_{y x}^{\mathrm{AH}} /\left[\rho_{x x}^{2}+\right.$ $\left.\rho_{y x}^{2}\right]$, where $\rho_{y x}^{\mathrm{AH}}$ represents the anomalous Hall resistivity, obtained through subtracting the normal Hall resisitivity $\rho_{y x}^{\mathrm{N}}\left(=R_{\mathrm{H}} H\right)$ from the measured $\rho_{y x}$. From Fig. 4(a), it can be seen that $\sigma_{x y}^{\mathrm{AH}}$ strongly depends on carrier type and density. Sample S1A, which is heavily electron doped, shows negative $\sigma_{x y}^{\mathrm{AH}}$, and its absolute value $\left|\sigma_{x y}^{\mathrm{AH}}\right|$ rises to a maximum of approximately $35 \Omega^{-1} \mathrm{~cm}^{-1}$ within the CAFM phase; we attribute these observations to the presence of an intrinsic AHE due to noncollinear spin structure [44]. However, in the FM phase, $\left|\sigma_{x y}^{\mathrm{AH}}\right|$ drops to approximately $18 \Omega^{-1} \mathrm{~cm}^{-1}$ and becomes nearly independent of the magnetic field. For the lightly electron-doped sample $S 3 A$, its $\sigma_{x y}^{\mathrm{AH}}$ shows not only a sign reversal from being positive to negative in the CAFM phase, but also quantum oscillations in the FM phase, with its nonoscillatory component decreasing with the increase of the magnetic field. In contrast, for heavily hole-doped samples, $\sigma_{x y}^{\mathrm{AH}}$ shows very small positive values (e.g., $\sigma_{x y}^{\mathrm{AH}} \sim 3 \Omega^{-1} \mathrm{~cm}^{-1}$ for sample $S 5 A$ ). However, when the hole carrier density is decreased to a moderate level, $\sigma_{x y}^{\mathrm{AH}}$ of the FM phase increases significantly. For instance, the maximal $\sigma_{x y}^{\mathrm{AH}}$ is approximately $38 \Omega^{-1} \mathrm{~cm}^{-1}$ for sample $S 4 A$, which is comparable to the theoretical value of $\sigma_{x y}^{\mathrm{AH}}$ estimated for the FM Weyl state using $\sigma_{x y}^{\mathrm{AH}}=\left(e^{2} / 2 \pi h\right) \Delta k$ [51], where $\Delta k$ represents the Weyl node separation $\left[\sigma_{x y}^{\mathrm{AH}} \sim 35 \Omega^{-1} \mathrm{~cm}^{-1}\right.$ (or $55 \Omega^{-1} \mathrm{~cm}^{-1}$ ) for the Weyl node separation of approximately $0.06 \AA^{-1}$ [36] (or $0.095 \AA^{-1}$ [37]) along the $k_{z}$ axis]. This result suggests that the relatively large $\sigma_{x y}^{\mathrm{AH}}$ observed in the moderately hole-doped samples should be of an intrinsic origin (see more discussions in Supplemental Material, Sec. S3 [48]). Note that sample $S 4 A$ also exhibits quantum oscillations in $\sigma_{x y}^{\mathrm{AH}}$ with its background showing a nearly linear decrease in the FM phase. This result can be attributed to the normal Hall contribution of a small electron pocket, which is not considered in the normal Hall subtraction. We consider only a single hole band contribution in the normal Hall subtraction for this sample. The inclusion of a negative normal Hall contribution of a small electron pocket in the $\sigma_{x y}^{\mathrm{AH}}$ extracted using $\sigma_{x y}^{\mathrm{AH}}=\rho_{y x}^{\mathrm{AH}} /\left[\rho_{x x}^{2}+\rho_{y x}^{2}\right]$ leads to an underestimated value of $\sigma_{x y}^{\mathrm{AH}}$. The intrinsic $\sigma_{x y}^{\mathrm{AH}}$ of sample
$S 4 A$ should be greater than $38 \Omega^{-1} \mathrm{~cm}^{-1}$. Similarly, the slight background increase of $\sigma_{x y}^{\mathrm{AH}}$ above $H_{c 2}$ for the lightly electron-doped sample $S 3 A$ should also be attributed to the coexistence of a large electron pocket with a small hole pocket, and the small positive normal Hall contribution from the small hole pocket is not considered in the normal Hall subtraction.

The coexistence of electron and hole pockets in the FM phase is more remarkably manifested by the temperature dependence of Hall conductivity in the lightly hole-doped samples $S 3 B$ and $S 3 C$ [Figs. 1(c) and 1(d)]. For these samples, it is difficult to separate $\rho_{y x}^{\mathrm{AH}}$ from $\rho_{y x}^{\mathrm{N}}$, since $\rho_{y x}^{\mathrm{N}}$ does not follow a linear field dependence at all due to the coexistence of electron and hole pockets [Figs. 1(c) and 1(d)]. For this reason, we attempt a different approach to evaluate $\sigma_{x y}^{\mathrm{AH}}$ for these samples. We compute the Hall conductivity at 2 and $24 \mathrm{~K}$ using $\sigma_{x y}=\rho_{y x} /\left(\rho_{x x}^{2}+\rho_{y x}^{2}\right)$. Since the magnetic transition temperatures of these samples are approximately $24.4 \mathrm{~K}$, we define the $\sigma_{x y}$ at $24 \mathrm{~K}$ as the normal Hall conductivity $\sigma_{x y}^{\mathrm{N}}$. If the PM-to-FM transition does not drive an electronic structure transition, the anomalous Hall conductivity $\sigma_{x y}^{\mathrm{AH}}$ at $2 \mathrm{~K}$ can be obtained by subtracting $\sigma_{x y}^{\mathrm{N}}$ from $\sigma_{x y}$ at $2 \mathrm{~K}$; this method is often used in analyzing the intrinsic anomalous Hall conductivity of magnetic topological semimetals (e.g., see Ref. [8]). As shown in Fig. 4(b), $\sigma_{x y}(2 \mathrm{~K})-\sigma_{x y}(24 \mathrm{~K})$ shows a strong nonlinear field dependence for samples $S 3 B$ and $S 3 C$; this result is inconsistent with the expected constant $\sigma_{x y}^{\mathrm{AH}}$ for the polarized FM phase but implies that the normal Hall contribution is not completely removed by subtracting $\sigma_{x y}(24 \mathrm{~K})$ from $\sigma_{x y}(2 \mathrm{~K})$ and undergoes a substantial change below the magnetic transition. This result not only provides further support for the argument that there is an electronic structure transition occurring concomitantly with the PM-to-FM magnetic transition near $24 \mathrm{~K}$ for the lightly hole-doped samples, but also suggests that the electronic structure varies with the temperature below $24 \mathrm{~K}$, which is indeed verified by the temperature dependence of the quantum oscillation frequency as presented below.

By contrast, for the heavily hole-doped sample $S 5 \mathrm{~A}$, which is not expected to exhibit an electronic structure transition driven by the magnetic transition, the value of $\left[\sigma_{x y}(2 \mathrm{~K})-\sigma_{x y}(24 \mathrm{~K})\right]$ indeed shows very weak field dependence and is comparable with the $\sigma_{x y}^{\mathrm{AH}}$ value obtained using $\sigma_{x y}^{\mathrm{AH}}=\rho_{y x}^{\mathrm{AH}} /\left[\rho_{x x}^{2}+\rho_{y x}^{2}\right]$, as shown in Fig. 4(b). Such a sharp contrast between the lightly and heavily hole-doped samples provides further support for the field-driven electronic structure reconstruction in the lightly hole-doped samples. Although it is difficult to separate $\sigma_{x y}^{\mathrm{AH}}$ from the normal Hall contribution for the lightly hole-doped samples, the fact that the experimentally extracted value of $\sigma_{x y}^{\mathrm{AH}}$ for the moderately hole-doped sample $S 4 A$ is comparable to the theoretical value expected for the ideal Weyl state as 
indicated above suggests that a large intrinsic AHE should also occur to the lightly hole-doped samples, since their Weyl nodes are closer to the Fermi level. Furthermore, the positive-to-negative sign reversal of $\left[\sigma_{x y}(2 \mathrm{~K})-\right.$ $\left.\sigma_{x y}(24 \mathrm{~K})\right]$ in the CAFM phases of samples $S 3 B$ and $S 3 C$ suggests that the field-driven electronic reconstruction should start to occur in the CAFM phases. The above interpretations for the field-dependent Hall conductivity shown in Figs. 4(a) and 4(b) are consistent with the evolution of Fermi surface predicted by theory for $\mathrm{Mn}\left(\mathrm{Bi}_{1-x} \mathrm{Sb}_{x}\right)_{2} \mathrm{Te}_{4}$ [52] - the coexistence of electron and hole pockets is present only when the chemical potential is close to the Weyl nodes, and the relative electron or hole pocket size is sensitively dependent on the chemical potential which is tuned by $\mathrm{Sb}$ concentration. Our DFT calculations reproduce similar results (see Supplemental Material, Sec. S4 and Fig. S11 [48]).

To further examine the evolution of the AHE with carrier density, we convert $\sigma_{x y}^{\mathrm{AH}}$ to anomalous Hall angle $\Theta_{x y}^{\mathrm{AH}}$ (see Sec. III and Supplemental Material, Fig. S3 [48]) and plot the maximal $\Theta_{x y}^{\mathrm{AH}}$ or $-\Theta_{x y}^{\mathrm{AH}}$ of the FM phase at $T=2 \mathrm{~K}$ as a function of the carrier density in Fig. 4(c). We do not include samples $S 3 B$ and $S 3 C$ in this plot, since their $\sigma_{x y}^{A H}$ cannot be separated from their normal Hall contribution. Figure 4(c) shows that $\left|\Theta_{x y}^{\mathrm{AH}}\right|$ increases with decreasing carrier density for both electron- and hole-doping regimes. For the hole-doping side, $\Theta_{x y}^{\mathrm{AH}}$ increases up to approximately $9 \%$ for moderately hole-doped samples. Since $\sigma_{x y}^{\mathrm{AH}}$ is slightly underestimated due to the residual normal Hall contribution of a small electron pocket for these samples as noted above, their intrinsic $\Theta_{x y}^{\mathrm{AH}}$ should be greater than $9 \%$.

The temperature dependences of $\rho_{x x}$ measured under magnetic fields for the lightly hole-doped samples also agree well for the expected field-induced FM WSM. As shown in Fig. 4(d), when the magnetic field is strong enough to drive the AFM state to the CAFM or FM state, $\rho_{x x}(T)$ exhibits an insulatinglike behavior, in contrast with the metallic behavior under zero magnetic field. Given that the magnetic-field-induced insulatinglike behavior is commonly observed in topological semimetals due to their large magnetoresistance at low temperatures [15], such behavior is consistent with the predicted field-induced FM WSM. We note that prior theoretical studies by Zhang et al. [36] suggest that this FM WSM can be either type I or type II depending on the lattice parameters. However, recent DFT calculations by Wang [53] suggest that in the $\mathrm{Mn}\left(\mathrm{Bi}_{1-x} \mathrm{Sb}_{x}\right)_{2} \mathrm{Te}_{4}$ alloy system, as the Sb content $x$ is increased from $x=0$ to 0.25 , a type-II Weyl state with the Weyl nodes being close to the Fermi level can be realized without the requirement of artificial lattice expansion, consistent with our experimental observations of the field-driven electronic structure transition in samples with $x$ being close to 0.25 . Our anomalous Hall conductivity data also point to a type-II WSM. If it were a type-I WSM, we would expect the sign of $\sigma_{x y}^{\mathrm{AH}}$ to always remains positive even when the system is tuned from hole doping to electron doping [51]. But, for a type-II WSM, theoretical studies [54] show that the sign of $\sigma_{x y}^{\mathrm{AH}}$ could vary from negative to positive when the chemical potential is tuned across the charge neutral point. Therefore, our observation of the sign reversal of anomalous Hall conductivity tuned by chemical potential [Fig. 4(a)] supports the conjecture that the FM WSM driven by the spin-flop transition in $\mathrm{Mn}\left(\mathrm{Bi}_{1-x} \mathrm{Sb}_{x}\right)_{2} \mathrm{Te}_{4}$ is a type-II WSM.

Another distinct magnetotransport property of a WSM is the chiral anomaly, which is manifested by negative longitudinal magnetoresistivity (LMR) [6,20,55,56]. Since the predicted FM WSM state for $\mathrm{MnBi}_{2} \mathrm{Te}_{4}$ has one pair of Weyl nodes aligned along the $k_{z}$ direction [36,37], its chiral anomaly is expected to be remarkable for the $c$-axis LMR. To probe such an effect, we perform $c$-axis magnetoresistivity $\mathrm{MR}=\left[\rho_{z z}(H)-\rho_{z z}(0)\right] / \rho_{z z}(0)$ measurements on both lightly and heavily hole-doped samples. Figure 5(a) presents the typical $c$-axis MR data of a lightly hole-doped sample $S 3 F$, which is cleaved from a piece with the measured carrier density of $n_{h}=0.097 \times 10^{20} \mathrm{~cm}^{-3}$. The spin-flop transition in this sample leads to a large negative LMR, with its magnitude rising to approximately $45 \%$ at $9 \mathrm{~T}$ and $2 \mathrm{~K}$. When the field is rotated away from the $c$ axis [inset, Fig. 5(b)], the magnitude of the MR gradually decreases and the sign of MR changes to positive when the field tilt angle is above $60^{\circ}$. In contrast, in a heavily holedoped sample $S 5 B\left(n_{h}=0.59 \times 10^{20} \mathrm{~cm}^{-3}\right)$, we find that the spin-flop transition leads to a relatively small $c$-axis negative LMR (MR -20\% at $2 \mathrm{~K}$ and $9 \mathrm{~T}$ ), and its MR hardly shows angular dependence in the polarized FM state above $4 \mathrm{~T}$ [see Fig. 5(b)], indicating that the negative MR of this sample originates from suppressed magnetic scattering, similar to the scenario seen in heavily electron-doped $\mathrm{MnBi}_{2} \mathrm{Te}_{4}$ [44]. The sharp contrast in the $c$-axis MR between the lightly hole-doped and heavily hole- and electron-doped samples suggests that the large negative LMR of sample $S 3 F$ should be dominated by the chiral anomaly. Of course, the negative MR component arising from suppressed magnetic scattering may not be completely excluded in this sample, but its contribution should be relatively small, as evidenced by the striking angular dependence of its MR [Fig. 5(a)]. The reason why only lightly hole-doped samples such as $S 3 F$ show signatures of chiral anomaly is that their Weyl nodes are close to the Fermi level, thus making the chiral anomaly effect significant. We also perform the in-plane LMR measurements on lightly hole-doped samples as well as the heavily electronand hole-doped samples, as presented in Fig. S4. The lightly hole-doped samples exhibit positive in-plane LMR, contrasted with the negative LMR due to the suppression of magnetic scattering probed in the heavily doped samples. The absence of in-plane LMR associated with the chiral anomaly in the lightly hole-doped samples can be attributed 


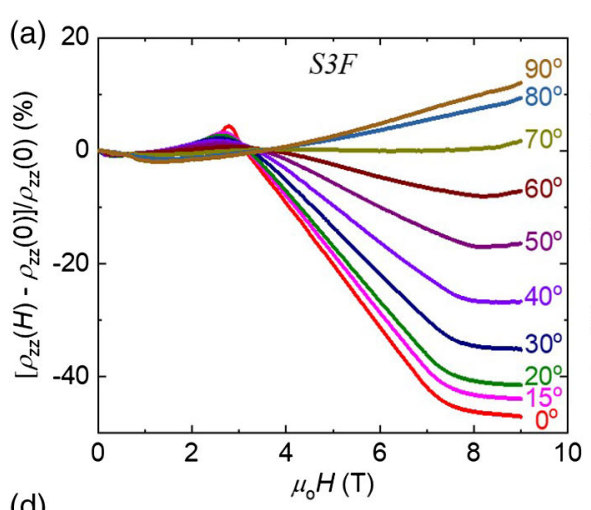

(d)

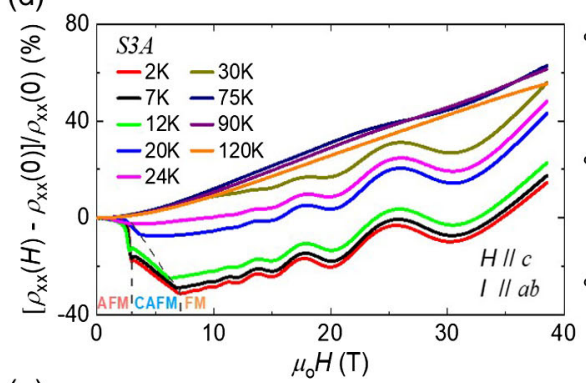

(g)

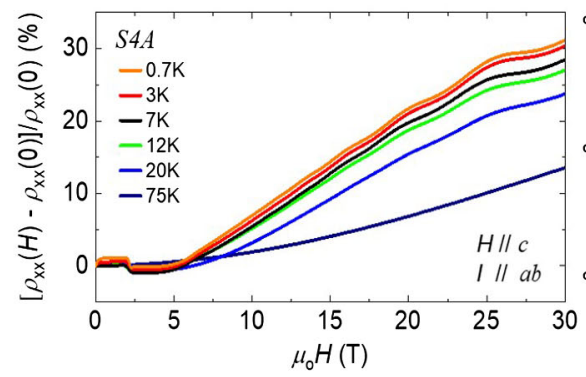

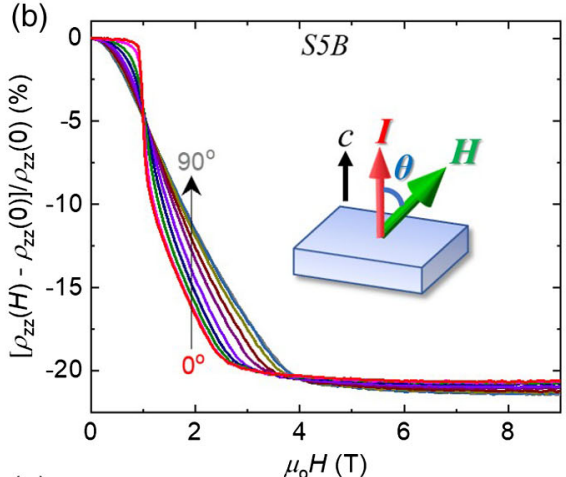

(e)

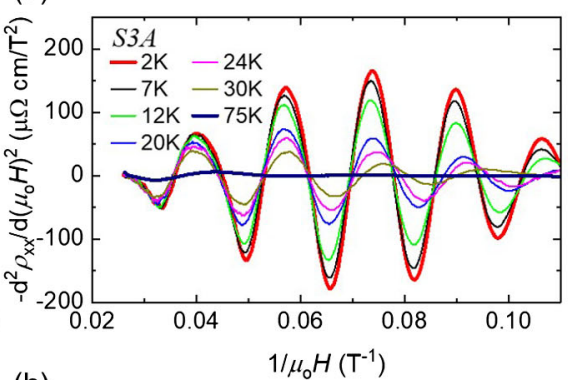

(h)

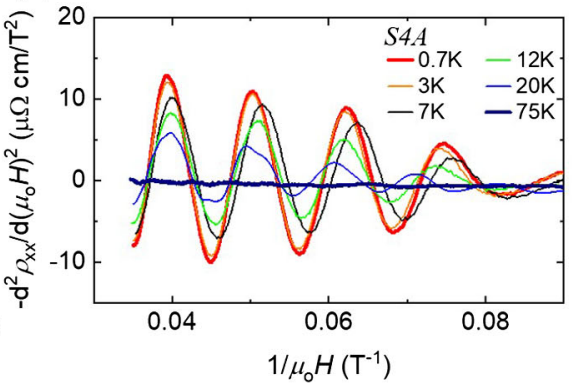

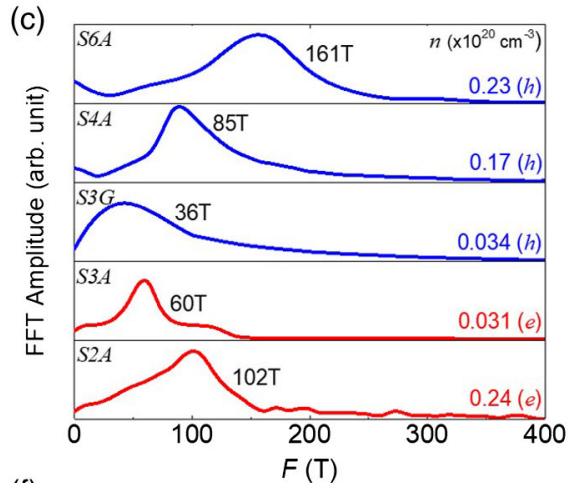

(f)

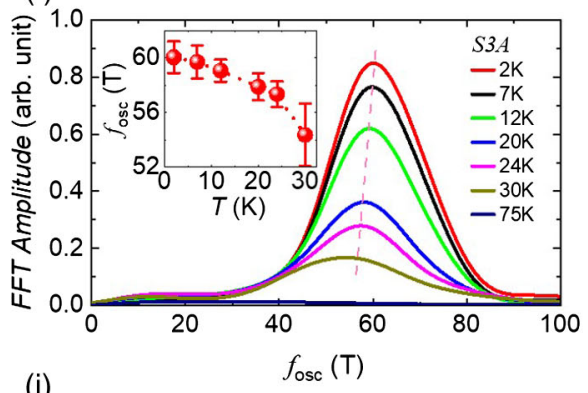

(i)

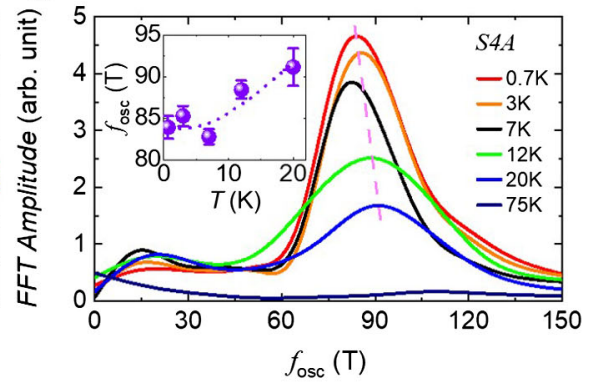

FIG. 5. Magnetoresistivity and $\mathrm{SdH}$ oscillations in $\mathrm{Mn}\left(\mathrm{Bi}_{1-x} \mathrm{Sb}_{x}\right)_{2} \mathrm{Te}_{4}$. (a),(b) The $c$-axis magnetoresistivity $\mathrm{MR}=\left[\rho_{z z}(H)-\right.$ $\left.\rho_{z z}(0)\right] / \rho_{z z}(0)$ under various field orientations for (a) the lightly $(S 3 F)$ and (b) heavily hole-doped $(S 5 B)$ samples. The schematic in (b) illustrates the experimental setup for the $c$-axis MR angular dependence measurements. (c) FFT spectra of $-d^{2} \rho_{x x} / d\left(\mu_{\mathrm{o}} H\right)^{2}$ for various moderately and lightly electron- and hole-doped samples [see Supplemental Material, Fig. S5(a) [48], for the SdH oscillations]. (d), (g) In-plane transverse MR of samples (d) $S 3 A$ and (g) $S 4 A$ at various temperatures. (e),(h) The second derivative of the in-plane resistivity $\rho_{x x}(H)$ with respect to field $H$ (perpendicular to the plane) for samples (e) $S 3 A$ and (h) $S 4 A$ as a function of the inverse of the field at various temperatures. (f),(i) FFT spectra of the second derivative of $\rho_{x x}(H)$ for samples (f) $S 3 A$ and (i) $S 4 A$ at various temperatures. The insets in both (f) and (i) show the oscillation frequency $\left(f_{\text {osc }}\right)$ as a function of the temperature for samples $S 3 A$ and $S 4 A$, respectively. The error bar shown in the insets in (f) and (i) is defined as the difference of the oscillation frequencies obtained from the FFT spectra of $-d^{2} \rho_{x x} / d\left(\mu_{\mathrm{o}} H\right)^{2}$ and $\Delta \rho_{x x}(H)$ [the oscillatory component of $\rho_{x x}(H)$ ], respectively. All the measurements shown in (c), (d), and (g) are conducted with the currents applied along the $a b$ plane and the magnetic fields perpendicular to the plane.

to the fact that the chiral anomaly of a type-II Weyl state is expected to be anisotropic, and the striking chiral anomaly is observed only when current is applied to specific directions [1,27].

The electronic structure evolution of $\mathrm{Mn}\left(\mathrm{Bi}_{1-x} \mathrm{Sb}_{x}\right)_{2} \mathrm{Te}_{4}$ with the magnetic transition is also corroborated by Shubnikov-de Haas ( $\mathrm{SdH}$ ) oscillations (see Supplemental Material, Fig. S5 [48]). SdH oscillation frequency decreases with carrier density for both electron and hole-doping sides [Fig. 5(c)]. However, the lightly hole-doped samples ( $S 3 B$ and $S 3 C$ ) do not exhibit $\mathrm{SdH}$ oscillations. This result is consistent with the picture established above, i.e., that the
Weyl nodes in the lightly hole-doped samples are close to the Fermi level. As shown in Figs. 5(d) and 5(g), the $\mathrm{SdH}$ oscillations of samples $S 3 A$ and $S 4 A$ appear following the spin-flop transitions, and they do not originate from topological surface states but stem from the bulk bands of the FM state (see more discussions in Supplemental Material, Sec. S5 [48]). The data in Fig. 5(d) also show that the SdH oscillations in sample $S 3 A$ extend to the PM state below $T=75 \mathrm{~K}$, where the applied magnetic field can drive the system to a forced FM state as noted above. From the second derivative of $\rho_{x x}(H)$ [Figs. 5(e) and 5(h)], it can be seen that the $\mathrm{SdH}$ oscillation pattern varies with the temperature in 
both samples. The oscillation frequency $f_{\text {osc }}$ derived from fast Fourier transform (FFT) analyses [Figs. 5(f) and 5(i)] increases by approximately $9.5 \%$ from 30 to $2 \mathrm{~K}$ for $S 3 \mathrm{~A}$ [inset, Fig. 5(f)] but decreases by approximately $8.8 \%$ from 20 to $0.7 \mathrm{~K}$ for $S 4 A$ [inset, Fig. 5(i)]. Such significant variations of $f_{\text {osc }}$ with the temperature suggest a strong coupling between the electronic structure and magnetism. As discussed above, the PM-to-FM transition in the lightly holedoped samples drives an electronic structure transition with the Fermi surface evolving from a hole pocket in the PM state to the one consisting of both electron and hole pockets. Such a Fermi surface reconstruction across the magnetic transition arises from the band splitting and crossing caused by the FM exchange interaction. While the FM exchange interactions sets in at the magnetic ordering temperature, the further decrease of temperature suppresses thermal fluctuations, thus enhancing the ordered moments, which should increase the band splitting. Therefore, both the electron and hole pocket sizes should vary with the temperature, which accounts for the temperature dependence of $f_{\text {osc }}$. The variation of the Fermi surface with the temperature for the FM phase of the lightly hole-doped samples is also manifested by the strong nonlinear field dependence of $\sigma_{x y}^{2 K}-\sigma_{x y}^{24 K}$ [Fig. 4(b)], as discussed above. For those samples showing $\mathrm{SdH}$ oscillations, we also estimate their carrier densities from $f_{\text {osc }}$ using $n_{\mathrm{SdH}}=n_{2 \mathrm{D}} / c$, where $n_{2 \mathrm{D}}=2 e f_{\text {osc }} / h, e$ is the elemental charge, $h$ is the Planck constant, and $c$ is the lattice parameter. As shown in Table I, $n_{\mathrm{SdH}}$ is comparable to the transport carrier density $n_{\text {Hall }}$ of the FM phase extracted from the Hall coefficient for the heavily electron- and hole-doped samples, but we cannot make this comparison for the lightly holedoped samples $S 3 B$ and $S 3 C$, since they do not show $\mathrm{SdH}$ oscillations. It is worth noting that for sample $S 4 A$, while its anomalous Hall conductivity data [Fig. 4(a)] suggest its Fermi surface of the FM phase involves the coexistence of a large hole pocket and a small electron pocket, only the hole pocket is probed in the $\mathrm{SdH}$ oscillations. The fact that the electron pocket is not manifested by the $\mathrm{SdH}$ oscillations can be attributed to the low electron mobility.

We also estimate the effective cyclotron mass $m_{c}^{*}$ through the fits of temperature dependences of the oscillation amplitude to the thermal damping factor of the LifshitzKosevich (LK) formula (see Supplemental Material, Fig. S5 [48]); $m_{c}^{*}$ is approximately $0.13 m_{\mathrm{o}}\left(m_{\mathrm{o}}\right.$ is the free electron mass) for sample $S 3 A$ and approximately $0.27 m_{\mathrm{o}}$ for sample $S 4 A$. The hole effective mass $m^{*}$ extracted from the band structure calculated using experimental lattice parameters at the density functional theory level (see Supplemental Material, Sec. S4 [48]) is $m^{*}=0.02 m_{\mathrm{o}}$ for the FM Weyl phase of $\mathrm{MnBi}_{2} \mathrm{Te}_{4}$, much smaller than that of the AFM phase, $m^{*}=0.33 m_{\mathrm{o}}$. For a calculated alloy structure of $\mathrm{Mn}\left(\mathrm{Bi}_{0.75} \mathrm{Sb}_{0.25}\right)_{2} \mathrm{Te}_{4}$ close to the experimental critical composition $(x=0.26)$, the hole effective mass extracted from the FM Weyl state is also about $0.02 m_{\mathrm{o}}$, increasing toward $0.1 m_{\mathrm{o}}$ at the boundaries of the plot, where the Fermi level is away from the Weyl point by approximately $60 \mathrm{meV}$ (see Supplemental Material, Sec. S4 [48]). This value is smaller than the experimentally measured $m^{*}=0.27 m_{\mathrm{o}}$ for sample $S 4 A$, which can be attributed to the fact that the Weyl nodes of this sample are farther from $E_{\mathrm{F}}$ than those of the samples with the composition of $\mathrm{Mn}\left(\mathrm{Bi}_{0.75} \mathrm{Sb}_{0.25}\right)_{2} \mathrm{Te}_{4}$.

We note that the layered compound $\mathrm{EuCd}_{2} \mathrm{As}_{2}$ with an $A$-type AFM order has also recently been reported to host a WSM state with one pair of Weyl nodes in either its PM state [57] or field-driven FM phase [58,59]. However, the nature of the Weyl state in $\mathrm{MnBi}_{2} \mathrm{Te}_{4}$ is quite distinct from that in $\mathrm{EuCd}_{2} \mathrm{As}_{2}$ : First, the Weyl cones in $\mathrm{MnBi}_{2} \mathrm{Te}_{4}$ are much more strongly tilted, forming a type-II WSM state; second, the tunability of the chemical potential by substituting $\mathrm{Sb}$ for Bi enables the observation of remarkable exotic quantum transport properties of Weyl fermions, including an intrinsic AHE and the chiral anomaly.

\section{METHODS}

\section{A. Sample preparation and measurements}

Single crystals of $\mathrm{Mn}\left(\mathrm{Bi}_{1-x} \mathrm{Sb}_{x}\right)_{2} \mathrm{Te}_{4}$ with $x$ from 0 to 1 are grown by a self-flux method as previously reported [50]. The mixtures of high-purity manganese powder (99.95\%), antimony shot (99.9999\%), bismuth shot (99.999\%), and tellurium ingot $(99.9999+\%)$ with the molar ratio of $\mathrm{Mn}: \mathrm{Sb}: \mathrm{Bi}: \mathrm{Te}=1: 5 x: 5(1-x): 16$ are loaded into an aluminum crucible and sealed in evacuated quartz tubes. The mixtures are heated up to $900^{\circ} \mathrm{C}$ for $12 \mathrm{~h}$ to promote homogeneous melting and slowly cooled down $\left(1.5^{\circ} \mathrm{C} / \mathrm{h}\right)$ to a temperature within the $590^{\circ} \mathrm{C}-630^{\circ} \mathrm{C}$ range (depending on $x$ ), followed by centrifugation to remove excess flux. Higher temperatures are used for the samples with higher $\mathrm{Sb}$ content to avoid the formation of $\mathrm{Sb}_{2} \mathrm{Te}_{3}$ (melting point approximately $620^{\circ} \mathrm{C}$ ). Soft, platelike single crystals up to $10 \times 5 \mathrm{~mm}^{2}$ can be obtained.

The phase purity of these single crystals is checked by $\mathrm{x}$ ray diffraction. As shown in Supplemental Material, Fig. S6 [48], the sharp $(00 L) \mathrm{x}$-ray diffraction peaks, which show only the small high-angle shift from $\mathrm{MnBi}_{2} \mathrm{Te}_{4}$ to $\mathrm{MnSb}_{2} \mathrm{Te}_{4}$, demonstrate the excellent crystallinity and the formation of the desired crystal structure with the stacking of Te-(Bi,Sb)-Te-Mn-Te-(Bi,Sb)-Te septuple layers in our single-crystal samples. The composition analyses by energy-dispersive x-ray spectroscopy (EDS) show the actual Sb content $x$ deviating from the nominal composition. In this article, we use the measured $\mathrm{Sb}$ content $x$.

The low magnetic field magnetotransport and magnetization measurements are performed using a commercial physical property measurement system (Quantum Design). The high magnetic field transport and magnetization measurements are carried out using the 31,35 , and 41.5 $\mathrm{T}$ resistive magnets at the NHMFL in Tallahassee. 
The standard four-probe method is used for magnetotransport measurements. Samples used for in-plane resistivity $\rho_{x x}$ and Hall resistivity $\rho_{y x}$ measurements have six attached leads, with one pair of current leads and two pairs of Hall voltage leads. The applied current is not aligned to any specific crystallographic axis in the $a b$ plane. Field sweeps of $\rho_{x x}$ and $\rho_{y x}$ are conducted for both positive and negative fields. The field dependences of $\rho_{x x}$ and $\rho_{y x}$ are obtained, respectively, using $\rho_{x x}=\left[\rho_{x x}\left(+\mu_{0} H\right)+\rho_{x x}\left(-\mu_{0} H\right)\right] / 2$ and $\rho_{y x}=\left[\rho_{y x}\left(+\mu_{\mathrm{o}} H\right)-\rho_{y x}\left(-\mu_{\mathrm{o}} H\right)\right] / 2$. The transport mobility $\mu_{\mathrm{H}}$ is inferred from the Hall coefficient $R_{\mathrm{H}}\{=$ $\left.\left[d \rho_{y x} / d\left(\mu_{\mathrm{o}} H\right)\right]\right\}$ via $\mu_{\mathrm{H}}=R_{\mathrm{H}} / \rho_{x x}(0)$, where $\rho_{x x}(0)$ is the zero-field longitudinal resistivity (shown in Supplemental Material, Fig. S2 [48]). The carrier density for the samples exhibiting linear field dependence in $\rho_{y x}$ is estimated via $n=\left(1 / e R_{\mathrm{H}}\right)$, where $e$ is the electric charge. For those samples showing nonlinear $\rho_{y x}(H)$, two-band model fitting is used to estimate the carrier density and its mobility (see Supplemental Material, Sec. S2 [48]). The VSM method is used for high field magnetization measurements. The ARPES measurements on sample $S 3 D$ are carried out using the 2DCC-MIP's ARPES facility at Penn State. The photoelectrons are excited by an unpolarized $\mathrm{He}-\mathrm{I}_{\alpha}$ light (21.218 eV), and two analyzers (Scientia R3000 and DA30L) are used for these measurements. The ARPES spectra of sample $S 3 E$ are acquired at the Advanced Light Source Maestro Beam line (BL 7.0.2) using the photon energy of $81 \mathrm{eV}$ and the microARPES end station. The energy resolution is approximately $19 \mathrm{meV}$, and the momentum resolution is $0.01 \AA^{-1}$.

\section{B. Analyses of anomalous Hall conductivity $\sigma_{x y}^{\mathrm{AH}}$ and anomalous Hall angle $\Theta_{x y}^{\mathrm{AH}}$}

We extract anomalous Hall conductivity $\sigma_{x y}^{\mathrm{AH}}$ from measured Hall resistivity $\rho_{y x}$ and longitudinal resistivity $\rho_{x x}$ via resistivity-to-conductivity tensor conversion. For the samples showing linear field dependence in $\rho_{y x}$ in their FM states (i.e., $S 1 A, S 2 A, S 3 A, S 4 A$, and $S 5 A$; see Fig. 1), their $\sigma_{x y}^{\mathrm{AH}}$ is derived using $\sigma_{x y}^{\mathrm{AH}}=\rho_{y x}^{\mathrm{AH}} /\left[\rho_{x x}^{2}+\rho_{y x}^{2}\right]$, where $\rho_{y x}^{\mathrm{AH}}$ is anomalous Hall resistivity, obtained after subtracting the normal Hall contribution $\left(\rho_{y x}^{\mathrm{N}}=R_{\mathrm{H}} H\right)$ from the measured $\rho_{y x}$. The $\rho_{x x}$ data of the samples shown in Fig. 1 are presented in Supplemental Material, Fig. S2 [48], except for the $x=0$ sample whose $\rho_{x x}$ data are reported in our previous work [44]. For those lightly doped samples, since their $\rho_{y x}$ of FM states do not show linear field dependence due to the coexistence of electron and hole pockets in the FM phase, we attempt a different approach to extract their anomalous Hall conductivity, i.e., $\sigma_{x y}^{\mathrm{AH}}=\sigma_{x y}-\sigma_{x y}^{\mathrm{N}}$ and $\sigma_{x y}=\rho_{y x} /\left(\rho_{x x}^{2}+\rho_{y x}^{2}\right)$. Here, $\sigma_{x y}^{\mathrm{N}}$ is the normal Hall conductivity, which can approximately be defined as the $\sigma_{x y}$ value at $24 \mathrm{~K}$, slightly below $T_{\mathrm{N}}(=24.4 \mathrm{~K})$. In Fig. S7 [48], we show the calculated $\sigma_{x y}$ of the $S 3 B$ sample as an example. As discussed in the main text, the anomalous Hall angle $\Theta_{x y}^{\mathrm{AH}}$ shown in Fig. 4(c) and Supplemental Material, Fig. S3 [48], is defined as $\sigma_{x y}^{\mathrm{AH}} / \sigma_{x x} ; \sigma_{x x}$ is also obtained via tensor conversion, i.e., $\sigma_{x x}=\rho_{x x} /\left(\rho_{x x}^{2}+\rho_{y x}^{2}\right)$.

\section{Band structure calculations}

All density functional theory calculations are performed using the Vienna ab initio simulation package (VASP) [60], using the generalized gradient approximation exchangecorrelation functional as parametrized by Perdew, Burke, and Ernzerhof $[61,62]$. Structural relaxations are performed with cutoff energies for plane-wave expansions at $400 \mathrm{eV}$, until forces are below $0.01 \mathrm{eV} / \AA$. Strong on-site Coulomb interactions of localized $3 d$ electrons are treated under the GGA $+U$ scheme, where the effective $U$ parameters $U_{\text {eff }}=U-J$ (in the approach of Ref. [63]) are $4 \mathrm{eV}$ for $3 d$ electrons in $\mathrm{Mn}$. Noncollinear magnetism and spin-orbit coupling are included following the VASP implementation [64]. Interlayer dispersion forces are described by the DFTD3 van der Waals correction scheme due to Grimme [65]. Following Ref. [43], the experimental lattice parameters we use are $a=4.334 \AA$ and $c=40.931 \AA$ for $\mathrm{MnBi}_{2} \mathrm{Te}_{4}$ and $a=4.310 \AA$ and $c=40.939 \AA$ for $\mathrm{Mn}\left(\mathrm{Bi}_{0.75} \mathrm{Sb}_{0.25}\right)_{2} \mathrm{Te}_{4}$.

\section{ACKNOWLEDGMENTS}

We thank C. X. Liu for helpful discussions. The study is based upon research conducted at The Pennsylvania State University Two-Dimensional Crystal ConsortiumMaterials Innovation Platform (2DCC-MIP), which is supported by National Science Foundation (NSF) Cooperative Agreement No. DMR-1539916. Z. Q. M. acknowledges the support from the U.S. National Science Foundation under Grant No. DM-1917579. J. H. acknowledges the U.S. Department of Energy (DOE), Office of Science, Basic Energy Sciences program under Grant No. DE-SC0019467 for the support of magnetization, quantum oscillation, and $31 \mathrm{~T}$ high field transport measurements. The work at the National High Magnetic Field Laboratory is supported by the NSF Cooperative Agreement No. DMR 1644779 and the State of Florida. L. M., J. H., and V. G.'s work is supported by the Penn State Center for Nanoscale Science, an NSF MRSEC under Grant No. DMR-2011839. C.Z. C., H. Y., and Y.F.Z. acknowledge the support from the DOE Grant (No. DESC0019064) and the NSF-CAREER grant (No. DMR1847811) for ARPES measurements at Penn State. The ARPES experiment at Berkeley was supported by the U.S. Department of Energy, Office of Science, Basic Energy Sciences, Materials Sciences and Engineering Division under Contract No. DE-AC02-05-CH11231 within the Quantum Material Program (KC2202). This experiment was performed by S. C. advised by A. L. and utilized the 
Advanced Light Source, a DOE Office of Science User Facility under Contract No. DE-AC02-05CH11231.

Note added.- -Recently, we became aware of a related work about $\mathrm{SdH}$ studies on the same material system [52].

[1] B. Yan and C. Felser, Topological Materials: Weyl Semimetals, Annu. Rev. Condens. Matter Phys. 8, 337 (2017).

[2] N. P. Armitage, E. J. Mele, and A. Vishwanath, Weyl and Dirac Semimetals in Three-Dimensional Solids, Rev. Mod. Phys. 90, 015001 (2018).

[3] A. Bernevig, H. Weng, Z. Fang, and X. Dai, Recent Progress in the Study of Topological Semimetals, J. Phys. Soc. Jpn. 87, 041001 (2018).

[4] G. Xu, H. Weng, Z. Wang, X. Dai, and Z. Fang, Chern Semimetal and the Quantized Anomalous Hall Effect in $\mathrm{HgCr}_{2} \mathrm{Se}_{4}$, Phys. Rev. Lett. 107, 186806 (2011).

[5] Q. Wang, Y. Xu, R. Lou, Z. Liu, M. Li, Y. Huang, D. Shen, $\mathrm{H}$. Weng, S. Wang, and H. Lei, Large Intrinsic Anomalous Hall Effect in Half-Metallic Ferromagnet $\mathrm{Co}_{3} \mathrm{Sn}_{2} \mathrm{~S}_{2}$ with Magnetic Weyl Fermions, Nat. Commun. 9, 3681 (2018).

[6] E. Liu et al., Giant Anomalous Hall Effect in a Ferromagnetic Kagome-Lattice Semimetal, Nat. Phys. 14, 1125 (2018).

[7] A. Sakai et al., Giant Anomalous Nernst Effect and Quantum-Critical Scaling in a Ferromagnetic Semimetal, Nat. Phys. 14, 1119 (2018).

[8] T. Suzuki, R. Chisnell, A. Devarakonda, Y. T. Liu, W. Feng, D. Xiao, J. W. Lynn, and J. G. Checkelsky, Large Anomalous Hall Effect in a Half-Heusler Antiferromagnet, Nat. Phys. 12, 1119 (2016).

[9] X. Wan, A. M. Turner, A. Vishwanath, and S. Y. Savrasov, Topological Semimetal and Fermi-Arc Surface States in the Electronic Structure of Pyrochlore Iridates, Phys. Rev. B 83, 205101 (2011).

[10] S.-Y. Xu et al., Discovery of a Weyl Fermion Semimetal and Topological Fermi Arcs, Science 349, 613 (2015).

[11] H. Weng, C. Fang, Z. Fang, B. A. Bernevig, and X. Dai, Weyl Semimetal Phase in Noncentrosymmetric TransitionMetal Monophosphides, Phys. Rev. X 5, 011029 (2015).

[12] A. C. Potter, I. Kimchi, and A. Vishwanath, Quantum Oscillations from Surface Fermi Arcs in Weyl and Dirac Semimetals, Nat. Commun. 5, 5161 (2014).

[13] P. J. W. Moll, N. L. Nair, T. Helm, A. C. Potter, I. Kimchi, A. Vishwanath, and J.G. Analytis, Transport Evidence for Fermi-Arc-Mediated Chirality Transfer in the Dirac Semimetal $\mathrm{Cd}_{3} \mathrm{As}_{2}$, Nature (London) 535, 266 (2016).

[14] C. Zhang et al., Quantum Hall Effect Based on Weyl Orbits in $\mathrm{Cd}_{3} \mathrm{As}_{2}$, Nature (London) 565, 331 (2019).

[15] J. Hu, S.-Y. Xu, N. Ni, and Z. Mao, Transport of Topological Semimetals, Annu. Rev. Mater. Res. 49, 207 (2019).

[16] B. Yang et al., Ideal Weyl Points and Helicoid Surface States in Artificial Photonic Crystal Structures, Science 359, 1013 (2018).

[17] S.-M. Huang et al., A Weyl Fermion Semimetal with Surface Fermi Arcs in the Transition Metal Monopnictide TaAs Class, Nat. Commun. 6, 7373 (2015).
[18] B. Q. Lv et al., Experimental Discovery of Weyl Semimetal TaAs, Phys. Rev. X 5, 031013 (2015).

[19] L.X. Yang et al., Weyl Semimetal Phase in the Noncentrosymmetric Compound TaAs, Nat. Phys. 11, 728 (2015).

[20] M. Hirschberger, S. Kushwaha, Z. Wang, Q. Gibson, S. Liang, C. A. Belvin, B. A. Bernevig, R. J. Cava, and N. P. Ong, The Chiral Anomaly and Thermopower of Weyl Fermions in the Half-Heusler GdPtBi, Nat. Mater. 15, 1161 (2016).

[21] Q. Xu, E. Liu, W. Shi, L. Muechler, J. Gayles, C. Felser, and Y. Sun, Topological Surface Fermi Arcs in the Magnetic Weyl Semimetal $\mathrm{Co}_{3} \mathrm{Sn}_{2} \mathrm{~S}_{2}$, Phys. Rev. B 97, 235416 (2018).

[22] D. F. Liu et al., Magnetic Weyl Semimetal Phase in a Kagomé Crystal, Science 365, 1282 (2019).

[23] J.-X. Yin et al., Negative Flat Band Magnetism in a SpinOrbit-Coupled Correlated Kagome Magnet, Nat. Phys. 15, 443 (2019).

[24] N. Morali, R. Batabyal, P. K. Nag, E. Liu, Q. Xu, Y. Sun, B. Yan, C. Felser, N. Avraham, and H. Beidenkopf, Fermi-Arc Diversity on Surface Terminations of the Magnetic Weyl Semimetal $\mathrm{Co}_{3} \mathrm{Sn}_{2} \mathrm{~S}_{2}$, Science 365, 1286 (2019).

[25] Z. Wang, M. G. Vergniory, S. Kushwaha, M. Hirschberger, E. V. Chulkov, A. Ernst, N. P. Ong, R. J. Cava, and B. A. Bernevig, Time-Reversal-Breaking Weyl Fermions in Magnetic Heusler Alloys, Phys. Rev. Lett. 117, 236401 (2016).

[26] I. Belopolski et al., Discovery of Topological Weyl Fermion Lines and Drumhead Surface States in a Room Temperature Magnet, Science 365, 1278 (2019).

[27] A. A. Soluyanov, D. Gresch, Z. Wang, Q. Wu, M. Troyer, X. Dai, and B. A. Bernevig, Type-II Weyl Semimetals, Nature (London) 527, 495 (2015).

[28] I. Belopolski et al., Discovery of a New Type of Topological Weyl Fermion Semimetal State in $\mathrm{Mo}_{x} \mathrm{~W}_{1-x} \mathrm{Te}_{2}$, Nat. Commun. 7, 13643 (2016).

[29] L. Huang et al., Spectroscopic Evidence for a Type II Weyl Semimetallic State in $\mathrm{MoTe}_{2}$, Nat. Mater. 15, 1155 (2016).

[30] K. Deng et al., Experimental Observation of Topological Fermi Arcs in Type-II Weyl Semimetal $\mathrm{MoTe}_{2}$ Nat. Phys. 12, 1105 (2016).

[31] S.-Y. Xu et al., Discovery of Lorentz-Violating Type II Weyl Fermions in LaAlGe, Sci. Adv. 3, e1603266 (2017).

[32] K. Koepernik, D. Kasinathan, D. V. Efremov, S. Khim, S. Borisenko, B. Büchner, and J. van den Brink, TaIrTe 4 : A Ternary Type-II Weyl Semimetal, Phys. Rev. B 93, 201101(R) (2016).

[33] I. Belopolski et al., Signatures of a Time-Reversal Symmetric Weyl Semimetal with Only Four Weyl Points, Nat. Commun. 8, 942 (2017).

[34] S. Borisenko et al., Time-Reversal Symmetry Breaking Type-II Weyl State in $\mathrm{YbMnBi}_{2}$, Nat. Commun. 10, 3424 (2019).

[35] J.-R. Soh et al., Magnetic Structure and Excitations of the Topological Semimetal $\mathrm{YbMnBi}_{2}$, Phys. Rev. B 100, 144431 (2019).

[36] D. Zhang, M. Shi, T. Zhu, D. Xing, H. Zhang, and J. Wang, Topological Axion States in the Magnetic Insulator $\mathrm{MnBi}_{2} \mathrm{Te}_{4}$ with the Quantized Magnetoelectric Effect, Phys. Rev. Lett. 122, 206401 (2019). 
[37] J. Li, Y. Li, S. Du, Z. Wang, B.-L. Gu, S.-C. Zhang, K. He, W. Duan, and Y. Xu, Intrinsic Magnetic Topological Insulators in van der Waals Layered $\mathrm{MnBi}_{2} \mathrm{Te}_{4}$-Family Materials, Sci. Adv. 5, eaaw5685 (2019).

[38] M. M. Otrokov et al., Prediction and Observation of an Antiferromagnetic Topological Insulator, Nature (London) 576, 416 (2019).

[39] Y. Deng, Y. Yu, M. Z. Shi, Z. Guo, Z. Xu, J. Wang, X. H. Chen, and Y. Zhang, Quantum Anomalous Hall Effect in Intrinsic Magnetic Topological Insulator $\mathrm{MnBi}_{2} \mathrm{Te}_{4}$, Science 367, 895 (2020).

[40] C. Liu, Y. Wang, H. Li, Y. Wu, Y. Li, J. Li, K. He, Y. Xu, J. Zhang, and Y. Wang, Robust Axion Insulator and Chern Insulator Phases in a Two-Dimensional Antiferromagnetic Topological Insulator, Nat. Mater. 19, 522 (2020).

[41] J. Ge, Y. Liu, J. Li, H. Li, T. Luo, Y. Wu, Y. Xu, and J. Wang, High-Chern-Number and High-Temperature Quantum Hall Effect without Landau Levels, Natl. Sci. Rev. 7, 1280 (2020).

[42] B. Chen et al., Intrinsic Magnetic Topological Insulator Phases in the $\mathrm{Sb}$ Doped $\mathrm{MnBi}_{2} \mathrm{Te}_{4}$ Bulks and Thin Flakes, Nat. Commun. 10, 4469 (2019).

[43] J. Q. Yan, S. Okamoto, M. A. McGuire, A. F. May, R. J. McQueeney, and B.C. Sales, Evolution of Structural, Magnetic, and Transport Properties in $\mathrm{MnBi}_{2-x} \mathrm{Sb}_{x} \mathrm{Te}_{4}$, Phys. Rev. B 100, 104409 (2019).

[44] S. H. Lee et al., Spin Scattering and Noncollinear Spin Structure-Induced Intrinsic Anomalous Hall Effect in Antiferromagnetic Topological Insulator $\mathrm{MnBi}_{2} \mathrm{Te}_{4}$, Phys. Rev. Research 1, 012011(R) (2019).

[45] T. Murakami, Y. Nambu, T. Koretsune, G. Xiangyu, T. Yamamoto, C. M. Brown, and H. Kageyama, Realization of Interlayer Ferromagnetic Interaction in $\mathrm{MnSb}_{2} \mathrm{Te}_{4}$ toward the Magnetic Weyl Semimetal State, Phys. Rev. B 100, 195103 (2019).

[46] Y. Chen et al., Ferromagnetism in van der Waals Compound $\mathrm{MnSb}_{1.8} \mathrm{Bi}_{0.2} \mathrm{Te}_{4}$, Phys. Rev. Mater. 4, 064411 (2020).

[47] Y. Liu et al., Site Mixing for Engineering Magnetic Topological Insulators, Phys. Rev. X 11, 021033 (2021).

[48] See Supplemental Material at http://link.aps.org/ supplemental/10.1103/PhysRevX.11.031032 for a detailed description.

[49] S. Ciocys, T. Morimoto, J. E. Moore, and A. Lanzara, Tracking Surface Photovoltage Dipole Geometry in $\mathrm{Bi}_{2} \mathrm{Se}_{3}$ with Time-Resolved Photoemission, J. Stat. Mech. 2019, 104008 (2019).

[50] J. Q. Yan, Q. Zhang, T. Heitmann, Z. Huang, K. Y. Chen, J.-G. Cheng, W. Wu, D. Vaknin, B.C. Sales, and
R. J. McQueeney, Crystal Growth and Magnetic Structure of $\mathrm{MnBi}_{2} \mathrm{Te}_{4}$, Phys. Rev. Mater. 3, 064202 (2019).

[51] A. A. Burkov, Anomalous Hall Effect in Weyl Metals, Phys. Rev. Lett. 113, 187202 (2014).

[52] Q. Jiang et al., Quantum Oscillations in the Field-Induced Ferromagnetic State of $\mathrm{MnBi}_{2-x} \mathrm{Sb}_{x} \mathrm{Te}_{4}$, Phys. Rev. B 103, 205111 (2021).

[53] Y. Wang, Chemical Requirements for Stabilizing Type-II Weyl Points in $\mathrm{MnBi}_{2-x} \mathrm{Sb}_{x} \mathrm{Te}_{4}$, arXiv:2103.12730.

[54] A. A. Zyuzin and R. P. Tiwari, Intrinsic Anomalous Hall Effect in Type-II Weyl Semimetals, JETP Lett. 103, 717 (2016).

[55] Y.-S. Jho and K.-S. Kim, Interplay between Interaction and Chiral Anomaly: Anisotropy in the Electrical Resistivity of Interacting Weyl Metals, Phys. Rev. B 87, 205133 (2013).

[56] D. T. Son and B. Z. Spivak, Chiral Anomaly and Classical Negative Magnetoresistance of Weyl Metals, Phys. Rev. B 88, 104412 (2013).

[57] J.-Z. Ma et al., Spin Fluctuation Induced Weyl Semimetal State in the Paramagnetic Phase of $\mathrm{EuCd}_{2} \mathrm{As}_{2}$, Sci. Adv. 5, eaaw4718 (2019).

[58] L.-L. Wang, N. H. Jo, B. Kuthanazhi, Y. Wu, R. J. McQueeney, A. Kaminski, and P. C. Canfield, Single Pair of Weyl Fermions in the Half-Metallic Semimetal $\mathrm{EuCd}_{2} \mathrm{As}_{2}$, Phys. Rev. B 99, 245147 (2019).

[59] J. R. Soh et al., Ideal Weyl Semimetal Induced by Magnetic Exchange, Phys. Rev. B 100, 201102(R) (2019).

[60] G. Kresse and J. Furthmüller, Efficient Iterative Schemes for Ab Initio Total-Energy Calculations Using a Plane-Wave Basis Set, Phys. Rev. B 54, 11169 (1996).

[61] J. P. Perdew, K. Burke, and M. Ernzerhof, Generalized Gradient Approximation Made Simple, Phys. Rev. Lett. 77, 3865 (1996).

[62] J. P. Perdew, K. Burke, and M. Ernzerhof, Phys. Rev. Lett. 78, 1396(E) (1997).

[63] S. L. Dudarev, G. A. Botton, S. Y. Savrasov, C. J. Humphreys, and A.P. Sutton, Electron-Energy-Loss Spectra and the Structural Stability of Nickel Oxide: An LSDA + U Study, Phys. Rev. B 57, 1505 (1998).

[64] D. Hobbs, G. Kresse, and J. Hafner, Fully Unconstrained Noncollinear Magnetism within the Projector AugmentedWave Method, Phys. Rev. B 62, 11556 (2000).

[65] S. Grimme, J. Antony, S. Ehrlich, and H. Krieg, A Consistent and Accurate Ab Initio Parametrization of Density Functional Dispersion Correction (DFT-D) for the 94 Elements H-Pu, J. Chem. Phys. 132, 154104 (2010). 